Draft Version June 21, 2021

Typeset using $\mathrm{LATEX}_{\mathrm{E}}$ default style in AASTeX63

\title{
Cloud Properties of Brown Dwarf Binaries Across the L/T Transition
}

\author{
Laci Shea Brock (D), ${ }^{1}$ Travis Barman (D), ${ }^{1}$ Quinn M. Konopacky (D), ${ }^{2}$ and Jordan M. Stone (D) 3 \\ ${ }^{1}$ Lunar and Planetary Laboratory 1629 E. University Blvd., Tucson, AZ 85721 USA \\ ${ }^{2}$ Center for Astrophysics and Space Sciences 9500 Gilman Dr., La Jolla, CA 92093 USA \\ ${ }^{3}$ Naval Research Laboratory, Remote Sensing Division, 4555 Overlook Ave SW, Washington, DC 20375 USA
}

(Received February 5, 2021; Revised April 26, 2021; Accepted April 26, 2021)

Submitted to ApJ

\begin{abstract}
We present a new suite of atmosphere models with flexible cloud parameters to investigate the effects of clouds on brown dwarfs across the $\mathrm{L} / \mathrm{T}$ transition. We fit these models to a sample of 13 objects with well-known masses, distances, and spectral types spanning L3-T5. Our modelling is guided by spatially-resolved photometry from the Hubble Space Telescope and the W. M. Keck Telescopes covering visible to near-infrared wavelengths. We find that, with appropriate cloud parameters, the data can be fit well by atmospheric models with temperature and surface gravity in agreement with the predictions of evolutionary models. We see a clear trend in the cloud parameters with spectral type, with earlier-type objects exhibiting higher-altitude clouds with smaller grains $(0.25-0.50 \mu \mathrm{m})$ and later-type objects being better fit with deeper clouds and larger grains $(\geq 1 \mu \mathrm{m})$. Our results confirm previous work that suggests $\mathrm{L}$ dwarfs are dominated by submicron particles, whereas $\mathrm{T}$ dwarfs have larger particle sizes.
\end{abstract}

Keywords: brown dwarfs: binaries, atmospheres, clouds

\section{INTRODUCTION}

As important opacity sources, clouds play a major role in determining the atmospheric structures, emergent spectra, and evolution of brown dwarfs. Clouds fundamentally impact the observational properties of color, magnitude, and spectra with compositions that span a diverse mix of condensates dependent upon a wide range of effective temperatures, gravities, metallicities, and pressures (Helling \& Casewell 2014; Marley \& Robinson 2015). The transition from $\mathrm{L}$ to $\mathrm{T}$ spectral types is perhaps the most well-known impact of changes in cloud opacity with observations of over 1000 field dwarfs highlighting the drastic shift in near-infrared colors from red to blue that occurs around a narrow range of effective temperatures ( $\approx 1400 \mathrm{~K}$ ) (Faherty et al. 2012; Dupuy \& Liu 2012; Leggett et al. 2002; Kirkpatrick 2005).

Atmospheres of L dwarfs are dominated by refractory materials condensing and forming optically thick cloud layers (e.g., Tsuji et al. 1996; Allard et al. 2001; Cushing et al. 2008; Marley et al. 2002). The common species include iron $(\mathrm{Fe})$, corundum $\left(\mathrm{Al}_{2} \mathrm{O}_{3}\right)$, enstatite $\left(\mathrm{MgSiO}_{3}\right)$, and forsterite $\left(\mathrm{Mg}_{2} \mathrm{SiO}_{4}\right)$. T dwarfs mark the disappearance of thick iron and silicate clouds from the photosphere and the appearance of methane absorption (Kirkpatrick 2005; Marley et al. 2010; Burgasser et al. 2003). Matching observed colors and spectra of L dwarfs requires some type of cloud layer in atmosphere models (Burrows et al. 2006). Mid-T dwarfs and cooler types are often best approximated using cloud-free atmosphere models (Kirkpatrick 2005); however, in some cases models with optically thin or inhomogeneous clouds composed of low temperature condensates $\left(\mathrm{Na}_{2} \mathrm{~S}, \mathrm{KCl}, \mathrm{ZnS}, \mathrm{MnS}, \mathrm{Cr}\right.$ ) can match T-dwarf photometry (Morley et al. 2012; Charnay et al. 2018).

Corresponding author: Laci S. Brock

laci@lpl.arizona.edu 
Self-consistent, one-dimensional atmosphere models have been the most widely used to study brown dwarfs. While they do not capture the atmospheric dynamics (e.g., Showman et al. 2018; Zhang \& Showman 2014; Tan \& Showman 2019, 2021a,b; Charnay et al. 2018) they do an excellent job of capturing the average properties and allow one to study the spectral trends across the brown dwarf population with a homogeneous set of modelling assumptions. The most recent model spectra have reached a high degree of realism through the development of molecular opacity databases (Freedman et al. 2008; Sharp \& Burrows 2007; Tennyson \& Yurchenko 2012), the chemistry of gas and condensate species (Lodders \& Fegley 2002), and resonance line broadening (Burrows et al. 2001; Allard et al. 2001). Incorporating parameterized clouds into atmosphere models better facilitates the interpretation of observational data, and these approaches have shown that $\mathrm{L}$ dwarfs and early $\mathrm{T}$ dwarfs are better represented by cloudy models rather than previous cloud-free models (Marley et al. 2002; Ackerman \& Marley 2001; Allard et al. 2001; Tsuji et al. 1996). In general, if appropriate input is provided, synthetic photometry, spectra, and colors generated by models are able to reproduce observational data quite well (Saumon \& Marley 2008).

Cloudy and cloud-free limits provide useful insight into the photometric and spectroscopic trends across brown dwarf spectral types, but these limiting cases do not accurately predict the scatter of colors within the L spectral type (Marley et al. 2010) and often fail to match individual brown dwarfs (Burrows et al. 2006). Liu et al. (2016) confirmed that lowgravity objects occupy a distinct region of the color-magnitude diagram separate from field brown dwarfs of the same spectral type. The complexities pose additional challenges in understanding how brown dwarfs evolve through time, especially across the $\mathrm{L} / \mathrm{T}$ transition( $\approx \mathrm{L} 8-\mathrm{T} 4)$, where major changes in atmospheric chemistry and dynamics occur. $\mathrm{L}$ dwarfs with red near-infrared colors are associated with signs of youth (Kirkpatrick et al. 2008; Cruz et al. 2009), low gravity, and/or high metallicity (McLean et al. 2003; Looper et al. 2008b; Stephens et al. 2009); whereas bluer L dwarfs are typically associated with an older age, higher gravity, and/or low metallicity (Schmidt et al. 2010; Burgasser et al. 2010; Cushing et al. 2010). Secondary influences from clouds can cause additional color dispersion, ranging from the thickness of the clouds to larger grain sizes (Knapp et al. 2004; Burgasser et al. 2008b). Although models can reproduce spectra of red L dwarfs with thick condensate clouds, there has been disagreement in effective temperatures across models (Gizis et al. 2012). The challenge is developing comprehensive atmosphere models while disentangling the effects of local cloud properties (e.g., thickness, grain size) within an atmosphere from global parameters (e.g., surface gravity, metallicity). Recent advancements use rotational modulation coupled with heterogeneous cloud models that include disequilibrium $\mathrm{CO} / \mathrm{CH}_{4}$ chemistry to isolate differences between cloud-induced and gravity-induced features (Lew et al. 2016, 2020).

Another approach to this problem is to investigate the properties of clouds in a sample of well-studied brown dwarf binary systems with precisely measured properties. Resolved photometry and spectroscopy for field age binaries of known distance and mass provide the clearest picture of the L-T spectral sequence as coevality can be assumed for such systems. The two components often have similar compositions, surface gravities, relatively constant radii for their age ( $\geq 0.5 \mathrm{Gyr}$ ), and tend to have nearly equal masses (Dupuy \& Liu 2012). Furthermore, individually resolved brown dwarf binaires provide the most robust tests for atmospheric and evolutionary models to-date through precisely measured dynamical masses. Mass is one of the most fundamental parameters of brown dwarfs that can aid in unravelling atmospheric complexities through its influence on surface gravity and evolution; however, mass is often difficult to measure and observations are complicated by the largely unknown span of ages for field objects due to degeneracies between mass and age (Konopacky et al. 2010; Dupuy \& Liu 2017).

Testing and constraining substellar evolutionary models requires benchmark brown dwarfs-objects with independently determined masses, luminosities, and ages. Early work hinted at a luminosity problem where comparisons between dynamical masses and masses predicted by evolutionary models differed significantly (Dupuy et al. 2009; Konopacky et al. 2010; Dupuy et al. 2014). The consistency between predicted and measured masses has improved with continued astrometric monitoring (Dupuy \& Liu 2017) and models that consider cloud clearing (Saumon \& Marley 2008; Allard 2013). A related problem exists for benchmark brown dwarfs when comparing the effective temperatures and gravities predicted by evolution models to those obtained by comparing model spectra to observed photometry or spectra. Differences in temperature as large as several hundred Kelvin and differences in gravity of an order of magnitude are not uncommon across the $\mathrm{L} / \mathrm{T}$ transition (Cushing et al. 2008). Clouds can alleviate some of the discrepancy between evolutionary and atmosphere model comparisons but does not eliminate the problem completely (Wood et al. 2019). Some of the issues may be improved with very broad wavelength coverage (Briesemeister et al. 2019). In addition to refined cloud parameters, metallicity (Wood et al. 2019; Crepp et al. 2018), non-equilibrium 
chemistry (Barman et al. 2011; Zhang et al. 2020), or other missing physics in the models (Charnay et al. 2018) (e.g., cloud microphysics) may be remaining sources of tension.

The importance of clouds shaping observed properties across the $\mathrm{L} / \mathrm{T}$ transition motivates our work to investigate cloud properties of individual brown dwarf binaries. An increase in the number of binary systems with known distance and mass across a broad range of temperatures allow us to explore the validity of previous discrepancies between predictions of atmosphere and evolutionary models.

In this paper, we test whether cloudy atmosphere models can be produced that match multi-band photometric observations to a reasonable degree. We focus on a sample of seven brown dwarf binary systems that span mid-L to mid- $\mathrm{T}$ in spectral type. By using a small sample size and a range of spectral types on either side of the $\mathrm{L} / \mathrm{T}$ transition, we are able to explore cloud properties in finer detail across a broad range of temperature and gravity. In addition to studying the cloud properties, we determine the effective temperatures for each binary component in the sample, independent of spectral type. Near-infrared photometry can be used to estimate $\mathrm{T}_{\text {eff }}$; however, because $\mathrm{T}_{\text {eff }}$ is a bolometric quantity, broad coverage of the spectral energy distribution (SED), especially on both sides of $\lambda_{\max }$, is highly desirable. We are able to determine a robust value of $\mathrm{T}_{\text {eff }}$ for these binaries for the first time using optical, spatially-resolved photometry provided by HST.

\section{BROWN DWARF BINARY SAMPLE}

\subsection{Sample Selection}

Approximately 68 visual, ultracool binaries (spectral type M7 or later) in the solar neighborhood have been identified in the last decade using high angular resolution imaging surveys conducted with the Hubble Space Telescope (HST) and ground-based adaptive optics systems (e.g., Lane et al. 2002; Liu et al. 2008; Burgasser et al. 2007). Over half of these binaries have been well-studied, undergoing extensive astrometric monitoring to determine precisely measured total and individual masses (30-115 $M_{\mathrm{Jup}}$ ) and robust orbits have been determined for 23 systems (Konopacky et al. 2010; Dupuy \& Liu 2017). For a discussion on the larger initial sample selection, see Burgasser et al. (2007) and for more information regarding astrometric monitoring see Konopacky et al. (2010) and Dupuy \& Liu (2017).

We selected seven binary systems from the set reported in Konopacky et al. (2010). These systems include 13 objects spanning spectral types from $\approx \mathrm{L} 4-\mathrm{T} 5$ and one late-M dwarf. Since the work published Konopacky et al. (2010), distance and mass values have been updated for many objects in our sample. A summary of properties from the literature and updated masses are provided in Table 1. Distance uncertainties have been improved for all objects in our sample. Table 2 shows previously used parallaxes with updated distance calculations based on work from Dupuy \& Liu (2017) and the recent GAIA DR2 release (Collaboration et al. 2018). 


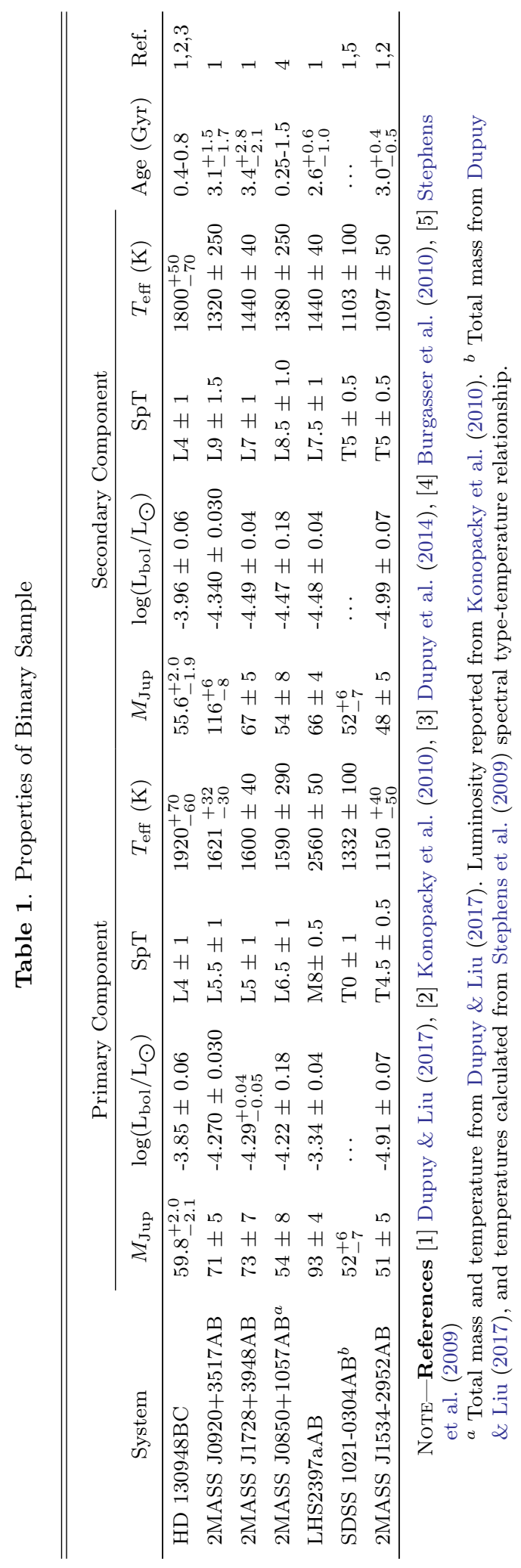


Table 2. Distances of Sample

\begin{tabular}{llllc}
\hline \hline \multicolumn{1}{c}{ Target } & Distance $[\mathrm{pc}]$ & \multicolumn{1}{c}{ Parallax $[\mathrm{mas}]$} & Updated Distance $[\mathrm{pc}]$ & $\Delta$ \\
\hline HD 130948BC & $18.18 \pm 0.08$ & $55.73 \pm 0.80$ & $17.94 \pm 0.25$ & $1 \%$ \\
2MASS 0920+35AB & $24.3 \pm 5.0$ & $32.3 \pm 0.6$ & $31.0 \pm 0.60$ & $28 \%$ \\
2MASS 1728+39AB & $24.1 \pm 2.1$ & $36.4 \pm 0.6$ & $27.5 \pm 0.47$ & $14 \%$ \\
2MASS 0850+10AB & $38.1 \pm 7.3$ & $31.4 \pm 0.6$ & $31.8 \pm 0.55$ & $17 \%$ \\
LHS 2397aAB & $14.3 \pm 0.4$ & $69.4903 \pm 0.1760$ & $14.3905 \pm 0.0364$ & $1 \%$ \\
SDSS 1021-03AB & $\cdots$ & $33.7 \pm 1.2$ & $29.7 \pm 1.05$ & $\cdots$ \\
2MASS 1534-29AB & $13.59 \pm 0.22$ & $63.0 \pm 1.1$ & $15.9 \pm 0.3$ & $15 \%$ \\
\hline
\end{tabular}

Note-We provide updated distances using the most recent parallax values from Collaboration et al. (2018) and Dupuy \& Liu (2017). A percent difference is calculated in column five to show improvement in precision. Note, SDSS 1021-03AB was not included in Konopacky et al. (2010).

\subsection{Photometry Measurements}

The years of astrometric monitoring of the brown dwarf binary components in our sample resulted in precise, spatially resolved $J, H$, and $K$ broad-band flux measurements. The majority of these near-infrared photometric measurements were obtained at the Keck Observatory using the NIRC2 instrument behind adaptive optics. Details on the observing and reduction procedure can be found in Konopacky et al. (2010).

To obtain photometry and astrometry, we used the package StarFinder from Diolaiti et al. (2000) (see Konopacky et al. 2010 for more details on the application for this dataset). For photometry, StarFinder provides the ratio of the fluxes of the binary components. We then use that ratio and the combined light magnitudes from 2MASS (Cutri et al. 2003) to derive individual apparent magnitude values for each component. Uncertainties are calculated by computing the RMS of the photometry from all individual images, and then propagating those uncertainties with those provided in the 2MASS catalog.

Additional photometric measurements were obtained from the Mikulski Archive for Space Telescopes (MAST). Most of the archival data are from programs 10559/PI:Bouy and 9451/PI:Brandner using Advanced Camera for Surveys (ACS) and filters F625W, F775W, F850LP. The archived data were supplemented with more recent observations using the Wide Field Camera 3 (WFC3/UVIS), 11605/PI:Barman. The filters (F625W, F775W, and F850LP) were chosen to provide Sloan-equivalent $r, i$, and $z$-band photometric measurements.

Data were collected in 2010 for Program 11605. We observed each target four times per filter. Both raw and calibrated data frames were retrieved post observation from MAST. Data are processed through the standard calibration pipeline for WFC3, as described in the WFC3 Data Handbook (Gennaro et al. 2019), including correction for geometric distortion.

Photometric measurements were obtained in two ways. First, we used the StarFinder algorithm to derive positions and fluxes of the components. We ran this algorithm on the distortion-corrected images. Since StarFinder requires a PSF estimate in the case of an uncrowded image like that of a binary star, we used TinyTim to generate synthetic PSFs at the proper wavelengths. Based on this PSF, StarFinder detected the components of the binary and returned a centroid and estimated flux. Based on those fluxes, we used the WFC3/UVIS zeropoints to compute the magnitudes of each source.

We also used the code written specifically for Hubble observations for photometry and astrometry, img2xym (Anderson \& King 2006), modified for WFC3/UVIS. It is designed to be used with the "FLT" images, on which distortion has not been applied. It outputs the positions and fluxes of stars that it identifies based on an isolation index which describes the allowed separation between sources. Running this code on our frames provided positions and fluxes, which were converted to magnitudes using the proper zeropoints.

Uncertainties in the WFC3/UVIS fluxes were determined as they were for NIRC2 data, by fitting all data frames individually and then looking at the RMS variation. We found that StarFinder and img2xym returned consistent fluxes, and hence magnitudes, for most cases. However, since a number of the binaries were very closely separated in 
Table 3. HST Photometric Observations

\begin{tabular}{|c|c|c|c|c|c|c|c|c|}
\hline $\begin{array}{c}\text { Target } \\
\text { Instrument }\end{array}$ & $\begin{array}{c}\text { F625W } \\
{[\mathrm{ACS}]}\end{array}$ & $\begin{array}{l}\text { F625W } \\
\text { [WFC3] }\end{array}$ & $\begin{array}{c}\text { F775W } \\
{[\text { ACS }]}\end{array}$ & $\begin{array}{l}\text { F775W } \\
\text { [WFC3] }\end{array}$ & $\begin{array}{c}\text { F814W } \\
{[\mathrm{WFPC}]}\end{array}$ & $\begin{array}{c}\text { F850LP } \\
{[\mathrm{ACS}]}\end{array}$ & $\begin{array}{l}\text { F850LP } \\
\text { [WFC3] }\end{array}$ & $\begin{array}{l}\text { F1042W } \\
\text { [WFPC2] }\end{array}$ \\
\hline 2 MASS $0920+35 \mathrm{~A}$ & $\cdots$ & $21.14 \pm 0.25$ & $\cdots$ & $18.35 \pm 0.12$ & $17.37 \pm 0.18$ & $\cdots$ & $15.75 \pm 0.18$ & $\cdots$ \\
\hline 2MASS $0920+35 B$ & $\ldots$ & $22.28 \pm 0.71$ & $\ldots$ & $19.36 \pm 0.36$ & $18.25 \pm 0.21$ & $\ldots$ & $16.07 \pm 0.15$ & $\ldots$ \\
\hline 2MASS $1728+39 \mathrm{~A}$ & $\cdots$ & $\ldots$ & $\cdots$ & $\ldots$ & $18.06 \pm 0.07$ & $\cdots$ & $\ldots$ & $15.60 \pm 0.10$ \\
\hline 2MASS $1728+39 \mathrm{~B}$ & $\ldots$ & $\ldots$ & $\ldots$ & $\ldots$ & $18.71 \pm 0.07$ & $\ldots$ & $\ldots$ & $15.35 \pm 0.12$ \\
\hline LHS 2397aA & $\cdots$ & $17.66 \pm 0.04$ & $\cdots$ & $15.75 \pm 0.05$ & $14.27 \pm 0.04$ & $\cdots$ & $12.63 \pm 0.05$ & $\ldots$ \\
\hline LHS 2397aB & $\cdots$ & $22.50 \pm 0.33$ & $\cdots$ & $20.60 \pm 0.34$ & $18.69 \pm 0.17$ & $\cdots$ & $16.78 \pm 0.09$ & $\cdots$ \\
\hline 2 MASS $0850+10 \mathrm{~A}$ & $21.32 \pm 0.28$ & $\ldots$ & $18.80 \pm 0.24$ & $\ldots$ & $17.78 \pm 0.15$ & $16.56 \pm 0.24$ & $\ldots$ & $\cdots$ \\
\hline 2 MASS $0850+10 B$ & $22.62 \pm 0.39$ & $\cdots$ & $19.96 \pm 0.28$ & $\ldots$ & $19.25 \pm 0.18$ & $17.42 \pm 0.26$ & $\ldots$ & $\cdots$ \\
\hline SDSS 1021-03A & $\ldots$ & $\cdots$ & $\ldots$ & $20.87 \pm 0.63$ & $\ldots$ & $\ldots$ & $17.01 \pm 0.62$ & $\cdots$ \\
\hline SDSS 1021-03B & $\cdots$ & $\cdots$ & $\cdots$ & $22.03 \pm 0.64$ & $\ldots$ & $\cdots$ & $17.42 \pm 0.62$ & $\ldots$ \\
\hline 2MASS $1534-29 \mathrm{~A}$ & $\ldots$ & $\cdots$ & $\cdots$ & $21.11 \pm 0.14$ & $19.22 \pm 0.05$ & $\cdots$ & $16.64 \pm 0.36$ & $15.39 \pm 0.12$ \\
\hline 2MASS 1534-29B & $\cdots$ & $\cdots$ & $\cdots$ & $21.61 \pm 0.20$ & $19.52 \pm 0.06$ & $\cdots$ & $17.04 \pm 0.15$ & $15.59 \pm 0.24$ \\
\hline
\end{tabular}

Table 4. Near-IR Photometric Observations

\begin{tabular}{lccc}
\hline \hline \multicolumn{1}{c}{ Target } & $J$ & $H$ & $K_{\mathrm{s}}$ \\
\hline HD 130948B & $12.53 \pm 0.07$ & $11.76 \pm 0.10$ & $10.98 \pm 0.04$ \\
HD 130948C & $12.84 \pm 0.08$ & $12.05 \pm 0.11$ & $11.18 \pm 0.04$ \\
2MASS 0920+35A & $13.89 \pm 0.17$ & $12.92 \pm 0.07$ & $12.12 \pm 0.08$ \\
2MASS 0920+35B & $13.94 \pm 0.33$ & $13.01 \pm 0.09$ & $12.44 \pm 0.10$ \\
2MASS 1728+39A & $14.27 \pm 0.09$ & $13.11 \pm 0.08$ & $12.20 \pm 0.06$ \\
2MASS 1728+39B & $14.58 \pm 0.09$ & $13.56 \pm 0.08$ & $12.80 \pm 0.06$ \\
LHS 2397aA & $11.18 \pm 0.02$ & $10.50 \pm 0.03$ & $10.02 \pm 0.02$ \\
LHS 2397aB & $14.65 \pm 0.08$ & $13.61 \pm 0.08$ & $12.79 \pm 0.04$ \\
2MASS 0850+10A & $14.24 \pm 0.07$ & $13.27 \pm 0.11$ & $12.56 \pm 0.10$ \\
2MASS 0850+10B & $14.70 \pm 0.14$ & $13.70 \pm 0.14$ & $12.91 \pm 0.16$ \\
SDSS 1021-03A & $14.22 \pm 0.09$ & $13.48 \pm 0.09$ & $13.27 \pm 0.08$ \\
SDSS 1021-03B & $14.33 \pm 0.09$ & $14.27 \pm 0.11$ & $14.25 \pm 0.08$ \\
2MASS 1534-29A & $14.57 \pm 0.08$ & $14.48 \pm 0.07$ & $14.46 \pm 0.13$ \\
2MASS 1534-29B & $14.73 \pm 0.12$ & $14.83 \pm 0.10$ & $14.73 \pm 0.15$ \\
\hline
\end{tabular}

the epoch of observation (e.g., 2MASS $0920+35 \mathrm{AB}$ was separated by $<1.5$ pixels), we opted to present here the results from the StarFinder analysis, as the img2xym code warns about unreliable results for stars separated by $<2$ pixels.

A complete list of photometry for our sample is provided in Tables 3 and 4 . In cases where the photometry was previously published, we have adopted those values here.

\section{SPECTRAL TYPE CLASSIFICATION}

We derive spectral types for the nine objects in our sample using template fits to the SEDs composed of resolved photometry for each object. We then compare our inferred spectral types to spectral types determined using spatially unresolved data and reported in the literature (Dupuy \& Liu 2012, 2017).

We complied a set of brown dwarf template spectra listed in Table 5 spanning spectral types M7 to T9 to compare to the objects in our sample. Both optical and near-IR spectral templates were used when available. Template spectra come from the Spex Prism Library collected by and maintained by Burgasser (2014). Each spectral template was absolute flux calibrated using 2MASS J-band photometry, and an absolute magnitude was determined for each brown 
Table 5. Spex Spectral Standards

\begin{tabular}{llc}
\hline \hline SpT & \multicolumn{1}{c}{ Template } & References \\
\hline M7 & VB 8 & {$[1]$} \\
M8 & VB 10 & {$[2]$} \\
M9 & LHS 2924 & {$[3]$} \\
L0 & 2MASS J0345+2540 & {$[3]$} \\
L1 & 2MASSW J1439+1929 (opt), 2MASSW J2130-0845 & {$[2,4]$} \\
L2 & 2MASSI J04082-1450 & {$[12]$} \\
L3 & 2MASSW J1146+2230 (opt), 2MASSW J1506+1321 & {$[5,6]$} \\
L4 & 2MASS J21580457-1550098 & {$[4]$} \\
L5 & DENIS-P J1228.2-1547 (opt), SDSS J083506.16+195304.4 & {$[5,7]$} \\
L6 & 2MASSs J0850+1057 (opt), 2MASSI J1010-0406 & {$[5,8]$} \\
L7 & DENIS-P J0205.4-1159 (opt), 2MASSI J0103+1935 & {$[5,9]$} \\
L8 & 2MASSW J1632+1904 & {$[6]$} \\
L9 & DENIS-P J0255-4700 & {$[10]$} \\
T0 & SDSS J120747.17+024424.8 & {$[11]$} \\
T1 & SDSS J015141.69+124429.6 & {$[2]$} \\
T2 & SDSSp J125453.90-012247.4 & {$[2]$} \\
T3 & 2MASS J1209-1004 & {$[2]$} \\
T4 & 2MASSI J2254188+312349 & {$[2]$} \\
T5 & 2MASS J15031961+2525196 & {$[2]$} \\
T6 & SDSSp J162414.37+002915.6 & {$[10]$} \\
T7 & 2MASSI J0727+1710 & {$[10]$} \\
T8 & 2MASSI J0415-0935 & {$[2]$} \\
T9 & UGPS J072227.51-054031.2 & {$[2]$} \\
\hline
\end{tabular}

NотE-A list of all brown dwarf templates used for spectral type determination obtained from the Spex Library. All templates had complete coverage from 0.8$2.5 \mu \mathrm{m}$. Additional optical spectral standards were included for L1, L3, L5, L6, and L7 types due to their availability in the library (denoted as opt).

References: [1] Burgasser et al. (2008a), [2] Burgasser et al. (2004), [3] Burgasser \& McElwain (2006), [4] Kirkpatrick et al. (2010), [5] Burgasser et al. (2010), [6] Burgasser et al. (2007), [7] Chiu et al. (2006), [8] Reid et al. (2006), [9] Cruz et al. (2004), [10] Burgasser et al. (2006), [11] Looper et al. (2008a), [12] Cruz et al. (2017)

dwarf template for seven of our band-pass filters for which there is complete spectral template coverage from 0.8-2.5 $\mu \mathrm{m}$.

$$
M=-2.5 \log \frac{\int f_{\lambda} S(\lambda) d \lambda}{\int f_{\text {Vega }} S(\lambda) d \lambda},
$$

where $f_{\lambda}$ and $f_{\text {Vega }}$ are the photon flux densities of the template spectrum and Vega, respectively, at 10 parsecs. $S(\lambda)$ is the filter response function.

We used a $\chi^{2}$ approach to find the best match

$$
\chi^{2}=\sum_{i}^{n} \frac{\left(M_{\mathrm{obs}, \mathrm{i}}-M_{\mathrm{Spex}, \mathrm{i}}+\delta M_{\mathrm{avg}}\right)^{2}}{\sigma_{\mathrm{obs}, \mathrm{i}}^{2}},
$$

where $n$ is the number of photometric measurements available for a given object, $\mathrm{M}_{\mathrm{obs}}$ are our observations, $\mathrm{M}_{\text {Spex }}$ are the Spex absolute magnitudes for each spectral template, $\delta \mathrm{M}_{\mathrm{avg}}$ is the average offset between the observations and Spex magnitudes, and $\sigma_{\text {obs }}$ are the uncertainties for our observations. The results of our fitting and resulting component spectral types are provided in Table 6 .

A lack of brown dwarf spectral templates with broad coverage across optical and near-infrared wavelengths limited our ability to use all of our HST data. However, the majority of spectral types we determined from our template 
Table 6. Spectral Type Fitting

\begin{tabular}{lcccc}
\hline \multicolumn{1}{c}{ Target } & SpT Lit. & Fit SpT & Fit Name & $\chi^{2}$ \\
\hline HD 130948B & L4 \pm 1 & L3 \pm 1 & 2MASSW J1146345+223053 & 3.3 \\
HD 130948C & L4 \pm 1 & L5 \pm 1 & SDSS J083506.16+195304.4 & 5.3 \\
2MASS 0920+35A & L5.5 \pm 1 & L3 \pm 3 & SDSS J083506.16+195304.4 & 4.5 \\
2MASS 0920+35B & L9 \pm 1.5 & L4 \pm 3 & DENIS-P J0205.4-1159 & 0.2 \\
2MASS 1728+39A & L5 \pm 1 & L7 \pm 1 & 2MASS J0103320+193536 & 5.4 \\
2MASS 1728+39B & L7 \pm 1 & L9 \pm 1 & DENIS-P J0255-4700 & 5.4 \\
LHS 2397aA & M8 \pm 1 & M8 \pm 1 & VB 10 & 5.60 \\
LHS 2397aB & L7.5 \pm 1 & L8 \pm 1 & 2MASSW J1632291+190441 & 2.1 \\
2MASS 0850+10A & L6.5 \pm 1 & L5 \pm 2 & SDSS J083506.16+195304.4 & 0.5 \\
2MASS 0850+10B & L8.5 \pm 1 & L6 \pm 2 & 2MASSs J0850359+105716 & 3.1 \\
SDSS 1021-03A & T0 \pm 1 & T2 \pm 1 & SDSSp J125453.90-012247.4 & 5.2 \\
SDSS 1021-03B & T5 \pm 1 & T8 \pm 1 & 2MASSI J0415195-093506 & 1.6 \\
2MASS 1534-29A & T4.5 \pm 1 & T5 \pm 1 & 2MASS J15031961+2525196 & 5.4 \\
2MASS 1534-29B & T5 \pm 1 & T6 \pm 1 & SDSSp J162414.37+002915.6 & 6.6 \\
\hline
\end{tabular}

Note-Previously determined spectral types for objects in our sample are listed in column two (Dupuy \& Liu 2017). Columns 3-4 provide the best-fitting brown dwarf template from Table 5. HD 130948B and 2MASS 0850+10B were both fit to optical templates for L3 and L6, respectively.

fitting were consistent with previously reported types from the literature. Table 6 shows a summary of our results. In a few cases for the coolest objects in our sample, we found a slightly later classification by 1-2 spectral types than Dupuy \& Liu (2017).

The 2MASS $0920+35 \mathrm{AB}$ system warrants further discussion. 2MASS $0920+35 \mathrm{~A}$ was fit to an earlier L3 \pm 3 but was consistent with literature (L5.5 \pm 1 ). 2MASS $0920+35 \mathrm{~B}$ was fit to L4 \pm 3 discrepant from the literature value by 0.5 (L9 \pm 1.5$)$. It was difficult to determine a type for both components in this system due to similar $\chi^{2}$ values for L3, L4, L5, L7 types. Dupuy \& Liu (2017) suggest 2MASS 0920+35B may be an unresolved binary partially due to a large individual mass of $116_{-8}^{+7} \mathrm{M}_{\mathrm{Jup}}$. The trends in spectral type and local minima further strengthen the possibility that 2 MASS $0920+35 \mathrm{~B}$ is indeed composed of more than one object.

The consistency of our fitted spectral types to the types reported in the literature give us confidence that our data, taken over multiple epochs and using multiple instruments, is sound. Given the large errors on some of our fitted spectral types; however, we will defer to the more precise types reported by Dupuy \& Liu (2017) whenever a spectral type is necessary.

\section{MODEL ATMOSPHERES}

To investigate the properties of the brown dwarfs in our sample further, we use the model atmosphere code PHOENIX to produce grids of synthetic spectra and photometry to compare to our data. We use the one-dimensional version of PHOENIX which self-consistently calculates the atmospheric structure and emergent spectrum under the assumptions of hydrostatic, chemical, and radiative-convective equilibrium (Hauschildt et al. 1998). PHOENIX solves the radiative transfer line-by-line and maintains a frequently updated database of molecular opacities (Tennyson \& Yurchenko 2012). The atmosphere models were calculated with a sampling of $1 \AA$ between 0.9 and $5 \mu \mathrm{m}$. For the remaining wavelength ranges, the sampling varies from 2 to $5 \AA$. For clarity, the model spectra have been convolved with a Gaussian of FWHM $\approx 70 \AA$ unless otherwise specified.

Solid and liquid particles suspended in an atmosphere (clouds) are arguably the most complex process to include in brown dwarf and giant planet atmosphere models. Atmospheres of substellar-mass objects span a large range of temperatures and pressures that allow the formation of a complex mixture of condensate species. Early works tackling the issues of cloud formation often focused on bracketing the extreme limits of condensate opacities by exploring cloudfree and complete chemical equilibrium clouds. Examples include the Cond and Dusty models of Allard et al. (2001) and comparable models from Burrows et al. (2001). These early works explained well the broad range of near-IR colors 
of brown dwarfs but were not intended to reproduce the observed properties of all objects, especially not those in or near the $\mathrm{L} / \mathrm{T}$ transition.

A number of cloud models with higher degrees of parameterization were developed to expand the applicability of atmosphere models for a greater variety of brown dwarfs suspected of containing clouds. Such models have included a range of tunable parameters for solid particle growth, mixing, and sedimentation timescales as well as mean grain size, particle size distributions, and explicit limits on position and vertical extent of the cloud (see Marley et al. (2002) or Helling et al. (2008b) for a comprehensive review). For this work we are interested in quantifying the simplest set of cloud parameters capable of reproducing the SEDs of brown dwarfs across the L/T transition. New atmosphere grids were developed specifically for this study informed by previous exoplanet atmosphere grids (Barman et al. 2011, 2015; Stone et al. 2020; Miles et al. 2020) and after extensive testing of various cloud properties.

To that end we use the parameterized cloud model described in Barman et al. (2011). The model includes one cloud composed of multiple condensates, each contributing to the total opacity based on their absorption and scattering cross-sections and relative number densities. A multiplicative weighting function is applied to the chemical equilibrium number densities where the value of the function is one for $\mathrm{P}_{\text {gas }}>\mathrm{P}_{c}$ and decreases exponentially for $\mathrm{P}_{\text {gas }} \leq \mathrm{P}_{c}$ (only a single $\mathrm{P}_{c}$ value is used for each atmosphere model). This weighting function is similar to the family of models described in Burrows et al. (2006); however, here the base of the cloud is always set by the deepest model layer where the chemical equilibrium condensate number density is non-zero. Figure 1 illustrates the basic structure for a model where $\mathrm{P}_{c}$ is larger than $\mathrm{P}_{g a s}$ at the cloud base. In such a situation, the condensate number densities across the cloud layers are multiplied only by the exponentially decaying part of the weighting function and, thus, are less than the chemical equilibrium values across all cloud layers. For models where $\mathrm{P}_{c}$ is smaller than $\mathrm{P}_{\text {gas }}$ at the cloud base, the condensate number densities will be equal to their chemical equilibrium values for layers between $\mathrm{P}_{c}$ and the cloud base, then dropping off for $\mathrm{P}_{g a s}<\mathrm{P}_{c}$. With this simple model, we can adjust the cloud's vertical extent and particle number density with a single parameter.

The cloud opacity is included self-consistently in the overall model calculation. Each model starts from a previous model calculation that is the closest to the new model across the various parameters. After each interation, the gas and condensate chemistry and associated opacities are recalculated based on a revised temperature structure. The process is repeated as the model converges toward radiative-convective equilibrium. Figure 2 illustrates how cloud pressure influences the spectrum and temperature-pressure profile.

For simplicity, grain size is assumed to be independent of height and governed by an additional free parameter of mean particle size, $a_{0}$. A log-normal distribution of grain sizes is included based on work from Marley et al. (1999). The range of mean particle sizes in our models span 0.25-10 $\mu \mathrm{m}$. Model grains include those in the PHOENIX database that are thermodynamically permissible and included in the total cloud opacity (Ferguson et al. 2005). Figure 3 illustrates how grain size influences the spectrum when $\mathrm{P}_{c}$ is held constant at 20 bars. At $1500 \mathrm{~K}$ adjustments to grain size have little impact at the $J$ band near $1.3 \mu \mathrm{m}$. However, as objects approach the $\mathrm{L} / \mathrm{T}$ transition and the temperature is decreased to $1300 \mathrm{~K}$, changes in $J$-band flux are more apparent and large differences in spectral shape can be seen between the $H$ and $K$ bands when comparing cloudy versus cloud-free models.

\section{RESULTS}

\subsection{Grid Fitting}

We fit resolved photometry to model grids to better understand the nature of clouds in substellar atmospheres spanning the L/T transition. Table 7 summarizes the grid properties and includes the new grids created specifically for this work, extending cloud properties across a broader range of pressures and incorporating models with smaller mean grain sizes. All models in the grid are solar metallicity with effective temperatures ranging from 800-2000 K and surface gravities of $\log (\mathrm{g})=3.0-5.5$. The conservative range of our models is suitable for objects from early L through early T dwarfs (Kirkpatrick 2005).

We calculate synthetic photometry for all models using HST and 2MASS filter transmission curves mentioned in Section 2. A best-fitting model is determined for each object using a $\chi^{2}$ fitting approach. We take the scale factor as

$$
s=(R / D)^{2},
$$

where $\mathrm{R}$ is the radius of the object to be fit, and $\mathrm{D}$ is 10 parsecs for absolute magnitude.

Table 8 shows the results of our model fitting. Models are allowed at the $68 \%$ confidence level by taking $\chi^{2}$ distributed with five degrees of freedom (the four atmosphere model parameters in Table 7 and the object's radius, 

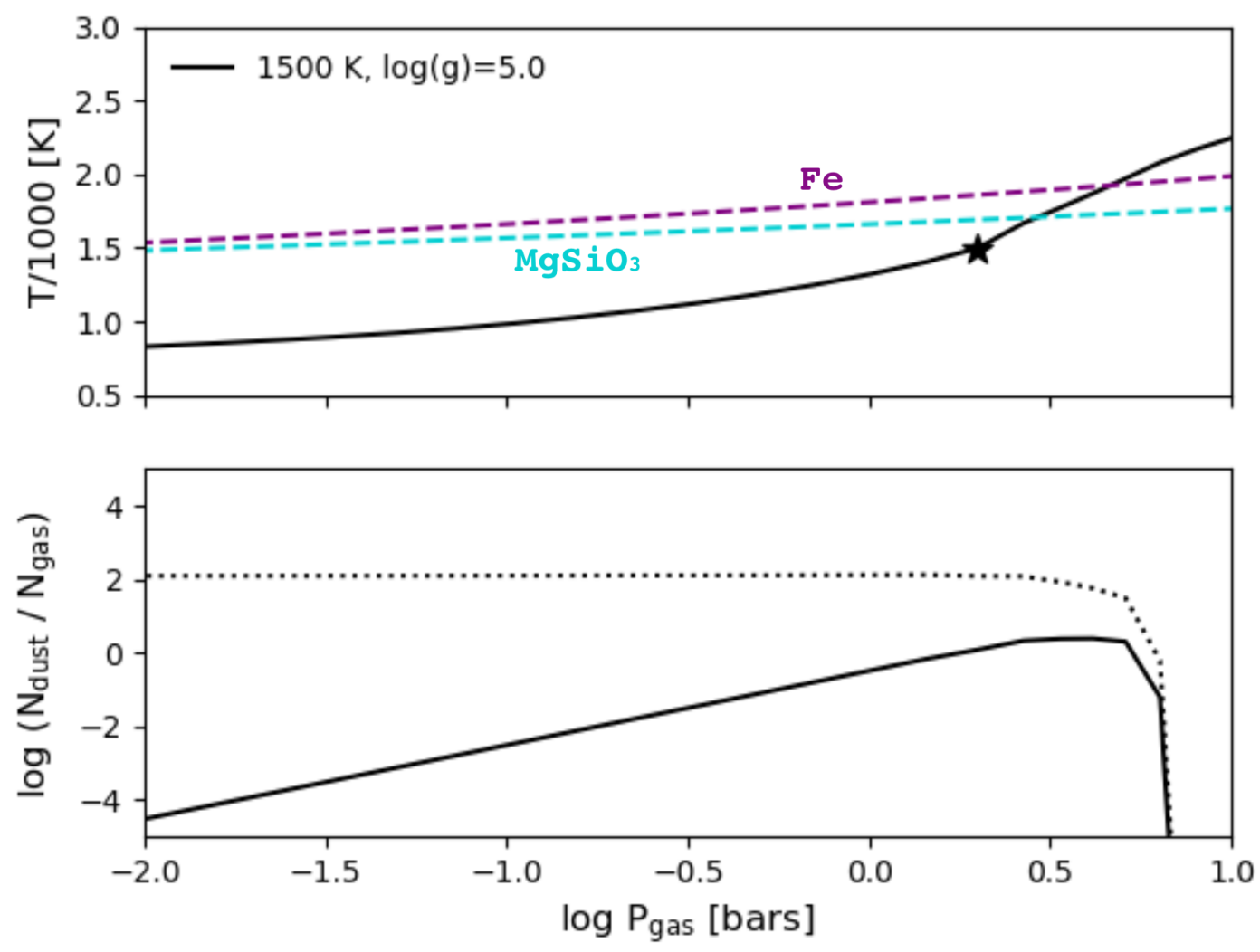

Figure 1. Top: Temperature-Pressure profile for cloudy atmosphere model with $\mathrm{P}_{c}=20$ bars and $\mathrm{a}_{0}=0.25 \mu \mathrm{m}$. Condensation curves for iron and enstatite are shown. The star represents the location of the photosphere. Bottom: dust-to-gas ratio for pure equilibrium clouds (dotted line) and intermediate cloudy model (solid line).

Table 7. Model Parameter Ranges

\begin{tabular}{lllll}
\hline \hline $\begin{array}{c}T_{\text {eff }} \\
{[\mathrm{K}]}\end{array}$ & \multicolumn{1}{c}{$\begin{array}{c}\log (\mathrm{g}) \\
{\left[\mathrm{cm} \mathrm{s}^{-2}\right]}\end{array}$} & \multicolumn{1}{c}{$\begin{array}{c}\mathrm{P}_{\mathrm{c}} \\
{[\mathrm{bar}]}\end{array}$} & \multicolumn{1}{c}{$\begin{array}{c}\mathrm{a}_{0} \\
{[\mu \mathrm{m}]}\end{array}$} & \multicolumn{1}{c}{ Increments } \\
\hline $800-2000$ & $3.5-5.5$ & $0.5,1,4$ & 1 & $100 \mathrm{~K} ; 0.5 \operatorname{in} \log (\mathrm{g})$ \\
$800-2000$ & $4.75,5.0,5.5$ & $10,20,30$ & $0.25,0.5,1$ & $100 \mathrm{~K}$ \\
$1700-2000$ & $4.5-5.5$ & $0.1,0.5$ & 0.25 & $100 \mathrm{~K} ; 0.5 \operatorname{in~} \log (\mathrm{g})$ \\
\hline
\end{tabular}

which is simultaneously fit). This includes all models with $\Delta \chi^{2}<11.3$. Listed first is the overall best-fitting model from the cloudy atmosphere grids. We then calculate the weighted mean parameters by

$$
\bar{m}=\frac{\Sigma_{i} W_{i} m_{i}}{\Sigma_{i} W_{i}}
$$

where the weight $W_{i}$ for each model $m_{i}$ is given by

$$
W_{i}=H_{i} e^{-0.5 \chi^{2}}
$$

where $H_{i}$ is a scaling factor that accounts for the uneven spacing of some atmospheric parameters on the grid, down weighting regions of dense sampling and up weighting regions of coarse sampling in proportion to the amount of parameter space covered. 

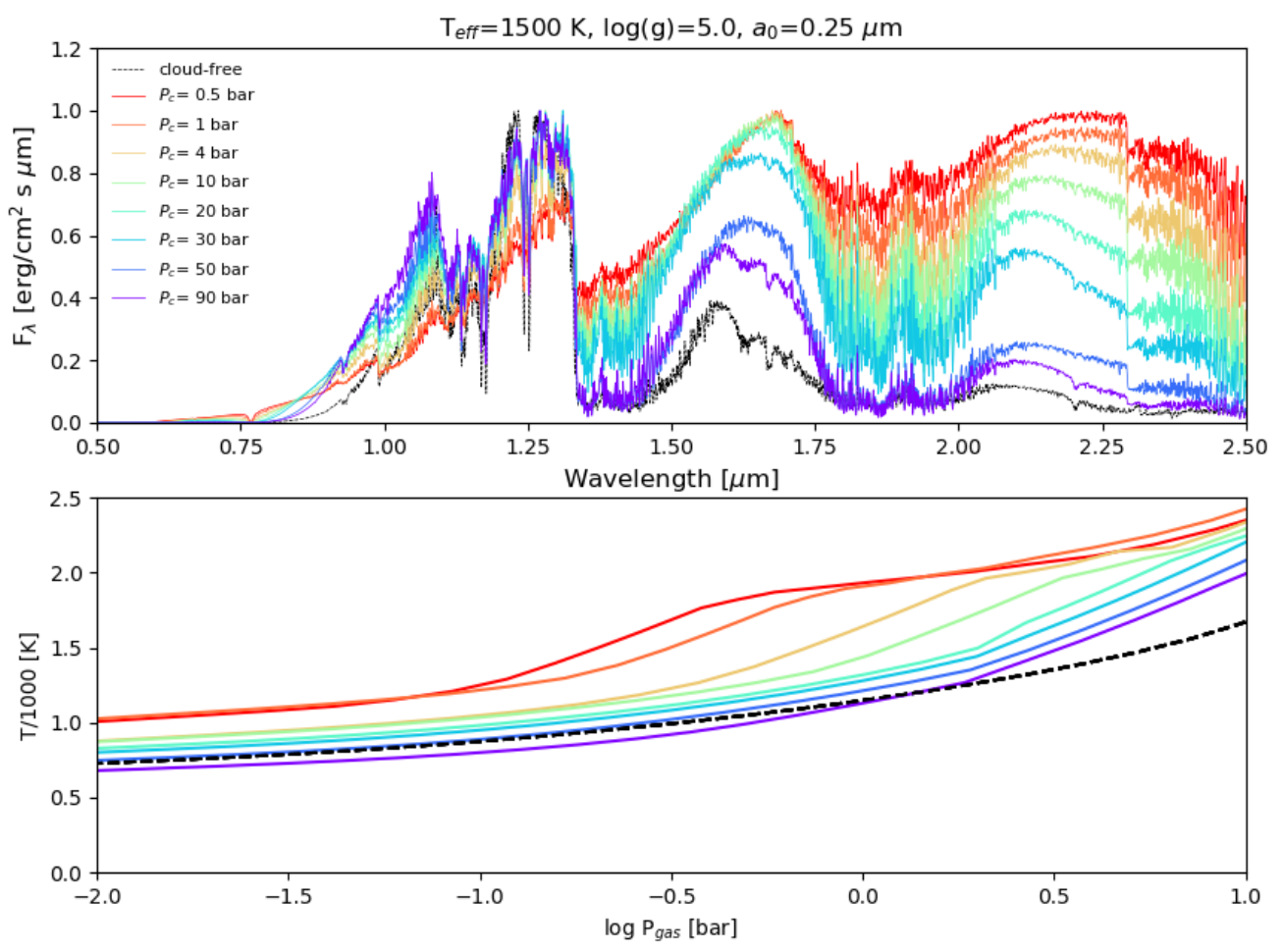

Figure 2. Comparison between model spectra and T-P profiles when cloud pressure, $\mathrm{P}_{c}$, is varied. A cloud-free model is provided in black. Spectra were normalized and convolved with a Gaussian of FWHM $\approx 12 \AA$ for clarity. All parameters are held constant except for cloud pressure.

We used the same approach as Stone et al. (2016) to calculate uncertainties using sided variance estimates with

$$
\sigma_{m}=\frac{\Sigma_{i} W_{i}\left(m_{i}-\bar{m}\right)^{2}}{\sum_{i} W_{i}}
$$

where the sum is calculated using parameters above $(+)$ or below $(-)$ the mean values. In cases where the edge of the grid boundary was approached, we report an upper/lower limit for our uncertainty.

The results of our analysis are shown in Figures 4 and 5 for our sample of objects. We plot the best-fit and weighted mean grid models for each object from Table 8. These fits highlight the role clouds play in the uncertainties of effective temperature, gravity, and radius when fitting cloudy model grids to photometric observations. Three objects, 2MASS $1728+39 \mathrm{AB}$ and LHS $2397 \mathrm{aB}$, were fit well by the grid and had properties consistent with evolutionary models shown in Figures 4 (c) and 5 (a).

Our fit of five parameters to HD 130948BC is not adequately constrained by the three photometric points used here. To help guide our fitting in subsequent sections and approach a unique solution for these objects, we applied a prior on radius (0.8-1.2 $\mathrm{R}_{\mathrm{Jup}}$ ) from evolutionary models for the weighted mean fits in Table 8 . The unconstrained best-fit model and weighted fit are provided in Figure 4 (a). This shows how two atmosphere models with very different effective temperatures, gravities, and cloud properties can match $J H K$ photometric observations well. The best fits plotted in purple are much cooler than expected for early L-type objects and approach non-physical radii $\left(\geq 1.5 R_{\text {Jup }}\right)$.

We note a few other objects where the grid fits present tension: 2MASS 0850+10AB, 2MASS 0920+35A, SDSS 1021-03AB, 2MASS 1534-29AB. Without incorporating evolutionary constraints on radius for the aforementioned systems, models allowed at 2- $\sigma$ over or under-predict effective temperature expected for a given spectral type (Leggett et al. 2002; Nakajima et al. 2004) and can result in non-physical radii. We believe this may be influenced by an unresolved component for some objects (e.g., 2MASS $0850+10 \mathrm{~A}$ ), and the cloud parameter space used in our grids does not appear to be appropriate for early-to-mid T dwarfs (e.g., 2MASS 1534-29AB). Figure 4 shows the grid fits 

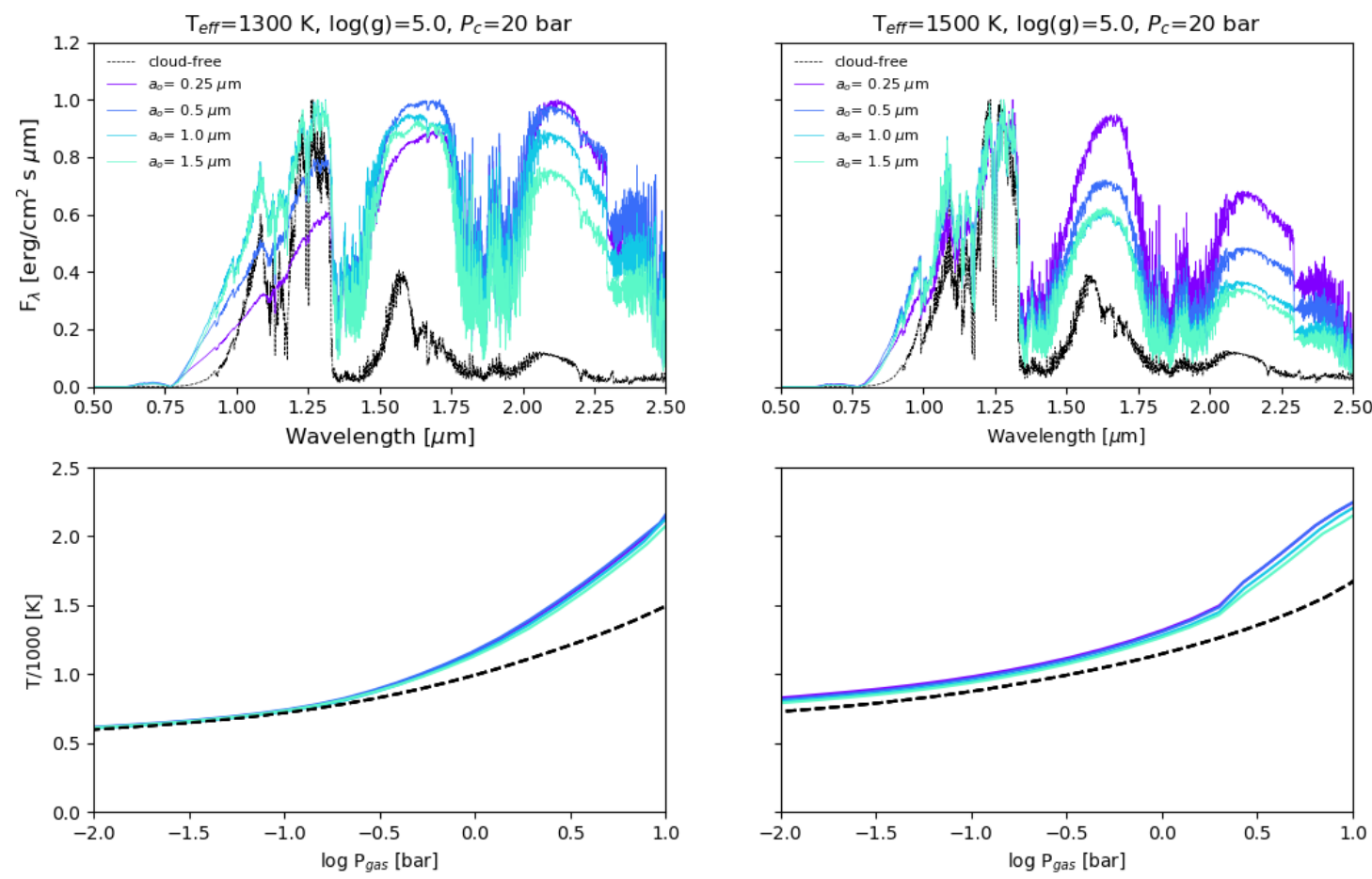

Figure 3. Comparison between model spectra and T-P profiles when mean grain size is varied. A cloud-free model is provided in black. Normalized spectra are shown for two effective temperatures. Spectra are convolved with a Gaussian of FWHM $\approx 12$ $\AA$ for clarity. All parameters held constant except for grain size in each panel.

Table 8. Model Atmosphere Fits

\begin{tabular}{|c|c|c|c|c|c|c|c|c|c|c|}
\hline \multirow[b]{2}{*}{ Name } & \multicolumn{5}{|c|}{ Best Fit } & \multicolumn{5}{|c|}{ Weighted Mean } \\
\hline & $\mathrm{T}_{\text {eff }}$ & $\log (g)$ & $\mathrm{R}_{\text {Jup }}$ & $\mathrm{P}_{\mathrm{c}}$ & $a_{0}$ & $\mathrm{~T}_{\text {eff }}$ & $\log (g)$ & $\mathrm{R}_{\mathrm{Jup}}$ & $\mathrm{P}_{\mathrm{c}}$ & $a_{0}$ \\
\hline HD 130948B & 1400 & 3.5 & 1.68 & 4 & 1.0 & $1903_{-4}^{+97}$ & $4.5_{-1.0}^{+0.50}$ & $1.04_{-0.29}^{+0.27}$ & $0.22_{-0.12}^{+0.28}$ & $\leq 1.0$ \\
\hline HD $130948 \mathrm{C}$ & 1400 & 4.0 & 1.76 & 4 & 1.0 & $1808_{-8}^{+92}$ & $4.5_{-1.0}^{+0.59}$ & $1.03_{-0.34}^{+0.16}$ & $0.47_{-0.67}^{+0.03}$ & $\leq 1.0$ \\
\hline 2 MASS $0920+35$ A & 1300 & 4.75 & 1.50 & 4 & 1.0 & $1517_{-194}^{+331}$ & $4.88_{-0.72}^{+0.47}$ & $1.41_{-0.47}^{+0.34}$ & $2.9_{-2.6}^{+2.9}$ & $0.70_{-0.43}^{+0.30}$ \\
\hline 2 MASS $1728+39 \mathrm{~A}$ & 1500 & 5.5 & 0.95 & 30 & 0.25 & $1487_{-131}^{+29}$ & $5.17_{-0.32}^{+0.33}$ & $0.97_{-0.05}^{+0.13}$ & $20_{-9}^{+10}$ & $0.26_{-0.01}^{+0.68}$ \\
\hline 2MASS $1728+39 \mathrm{~B}$ & 1400 & 4.75 & 0.83 & 20 & 1.0 & $1420_{-22}^{+100}$ & $4.99_{-0.83}^{+0.43}$ & $0.89_{-0.09}^{+0.06}$ & $8_{-5}^{+10}$ & $0.93_{-0.44}^{+0.07}$ \\
\hline LHS2397aB & 1300 & 4.75 & 0.95 & 30 & 1.0 & $1413_{-102}^{+93}$ & $4.84_{-0.09}^{+0.23}$ & $0.81_{-0.11}^{+0.12}$ & $23_{-3}^{+7}$ & $0.54_{-0.27}^{+0.46}$ \\
\hline 2 MASS $0850+10 \mathrm{~A}$ & 1800 & 5.5 & 0.56 & 10 & 0.25 & $1717_{-207}^{+91}$ & $5.08_{-0.63}^{+0.42}$ & $0.65_{-0.10}^{+0.22}$ & $7.2_{-6.1}^{+4.2}$ & $0.36_{-0.11}^{+0.55}$ \\
\hline 2 MASS $0850+10 B$ & 1500 & 5.5 & 0.74 & 20 & 0.25 & $1423_{-277}^{+80}$ & $5.3_{-0.69}^{+0.20}$ & $0.93_{-0.18}^{+0.80}$ & $14_{-7}^{+10}$ & $0.37_{-0.12}^{+0.63}$ \\
\hline SDSS 1021-03A & 1700 & 5.0 & 0.50 & 30 & 0.25 & $1668_{-97}^{+37}$ & $4.92_{-0.17}^{+0.09}$ & $0.52_{-0.03}^{+0.09}$ & $28_{-8}^{+20}$ & $0.36_{-0.11}^{+0.57}$ \\
\hline SDSS 1021-03B & 1700 & 4.75 & 0.36 & 30 & 1.0 & $1698_{-98}^{+2}$ & $\leq 5.5$ & $0.36_{-0.02}^{+0.06}$ & $\geq 0.1$ & $0.96_{-0.57}^{+0.04}$ \\
\hline 2MASS $1534-29 \mathrm{~A}$ & 1700 & 5.5 & 0.38 & 30 & 0.25 & $1699_{-99}^{+1}$ & $\geq 3.5$ & $0.38_{-0.01}^{+0.02}$ & $24_{-4}^{+7}$ & $\leq 1.0$ \\
\hline 2MASS 1534-29B & 1700 & 5.5 & 0.32 & 20 & 0.25 & $1699_{-99}^{+1}$ & $\geq 3.5$ & $0.33 \pm 0.01$ & $21_{-3}^{+9}$ & $\leq 1.0$ \\
\hline
\end{tabular}

Noте-Best-fitting grid models and weighted means with 2- $\sigma$ uncertainties are provided. Weighted mean fits for HD $130948 \mathrm{BC}$ use a fixed range for radius 0.8-1.2 $\mathrm{R}_{\text {Jup }}$ constrained by Saumon \& Marley (2008) evolutionary models. Fits in the table for 2MASS 1728+39B exclude the F814W photometric point and are discussed further in Section 6.3.

for 2MASS $0850+10 \mathrm{AB}$, SDSS 1021-03AB, and 2MASS 1534-29AB are warmer ( $T_{\text {eff }} \geq 1700 \mathrm{~K}$ ) and approach smaller than expected radii ( $\leq 0.70 R_{\text {Jup }}$ ) for objects of field age (Dupuy \& Liu 2017). Conversely, the model fits for 2MASS $0920+35 \mathrm{~A}$ shown in Figure 5 have radii larger than expected $\left(\geq 1.4 R_{\mathrm{Jup}}\right)$ for mid-L dwarfs (Leggett et al. 2002; Nakajima et al. 2004). 


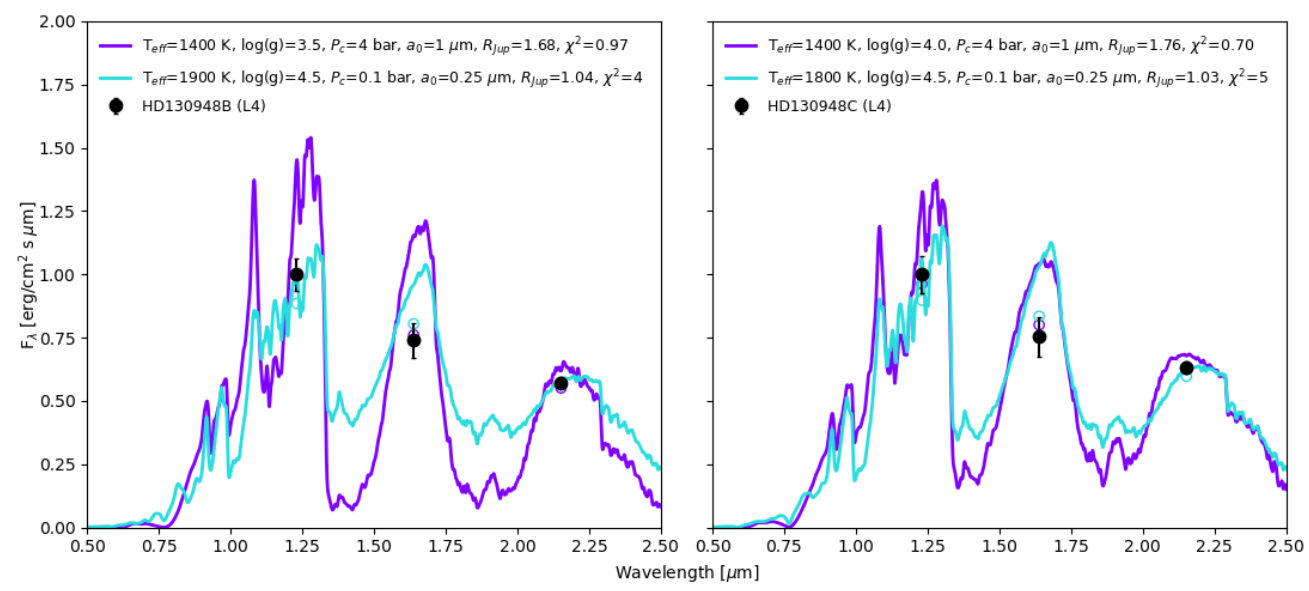

(a)

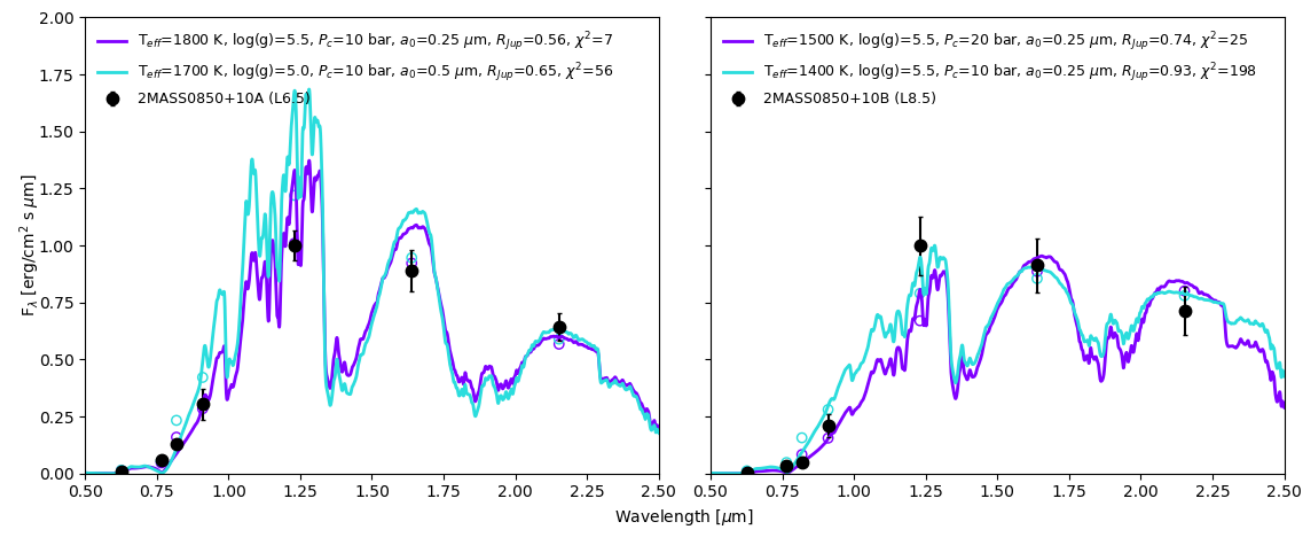

(b)

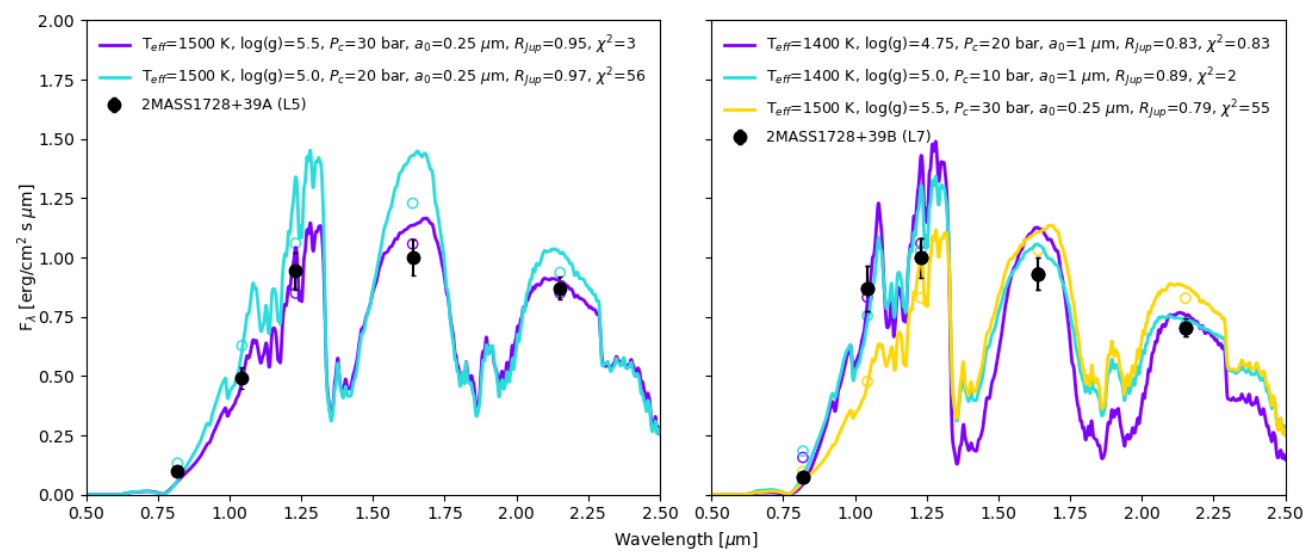

(c)

Figure 4. Best-fit (purple) and weighted mean (cyan) atmosphere models from Table 8. We chose the closest available model from our grid to the mean parameters. Corresponding synthetic photometry is plotted in same color as model spectra. Surface gravity is in $\mathrm{cm} \mathrm{s}^{-2}$. Spectral types included are from Dupuy \& Liu (2017). 


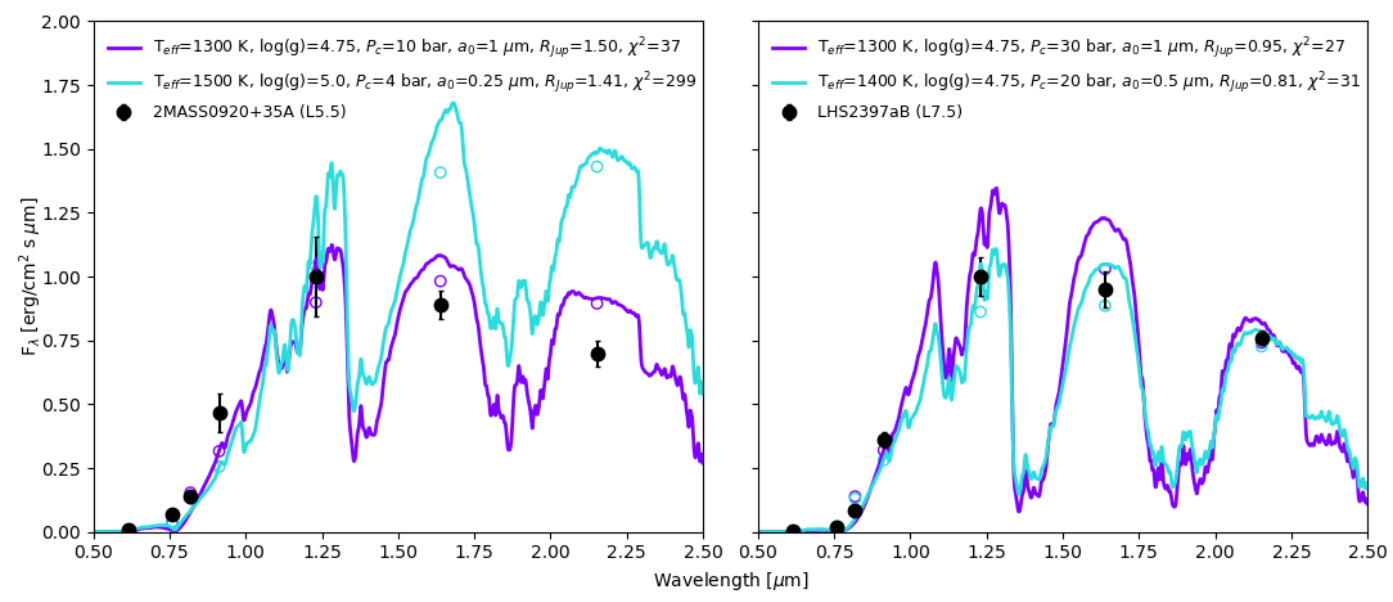

(a)

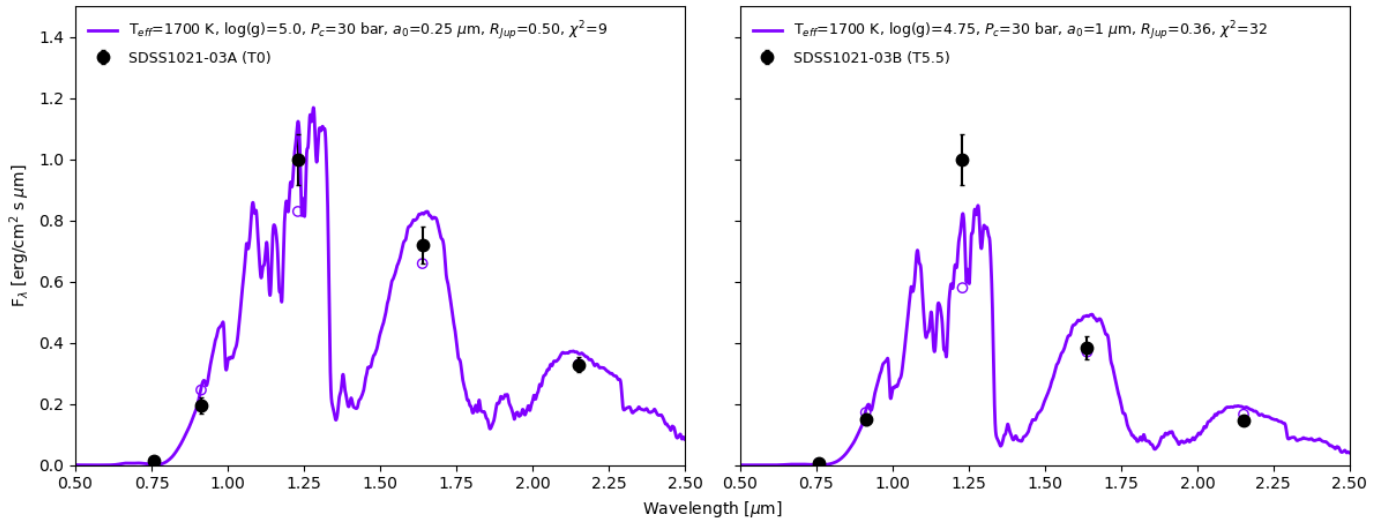

(b)

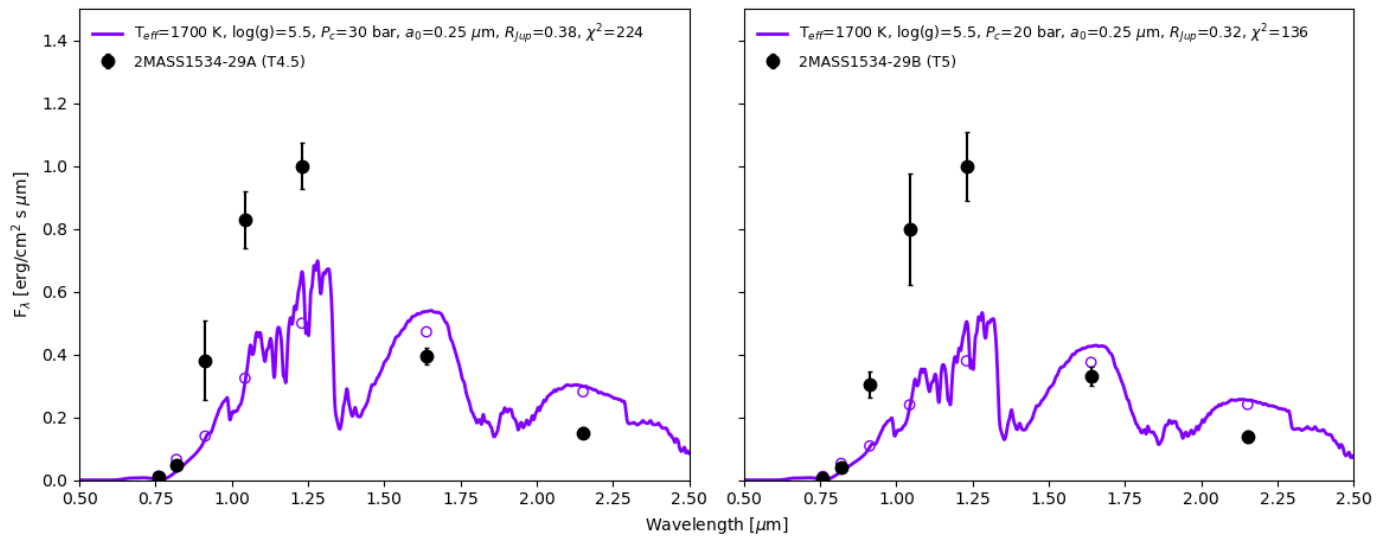

(c)

Figure 5. Best-fit (purple) and weighted mean (cyan) atmosphere models from Table 8. Only the best fits are shown for SDSS 1021-03AB and 2MASS 1534-29AB because weighted mean parameters are similar. Surface gravity is in $\mathrm{cm} s^{-2}$. Spectral types included are from Dupuy \& Liu (2017) 


\section{EVOLUTIONARY MODEL COMPARISONS}

The addition of cloud properties in atmosphere models can lead to further degeneracies in mass and radius resulting in good fits to photometric data but producing non-physical properties given what we know of brown dwarf physics. An effective way to evaluate the quality of atmosphere model fits is by comparing the results to the bulk properties from evolutionary model predictions. We use bolometric luminosity constrained from our grid fits combined with the measured masses (Dupuy \& Liu 2017; Konopacky et al. 2010) to derive evolutionary predictions. We derive evolutionary predictions using Saumon \& Marley (2008) hybrid grids for mid-to-late L dwarfs in our sample and use the Cond grids from Baraffe et al. (2003) for $\mathrm{T}$ dwarfs with $\mathrm{T}_{\text {eff }} \lesssim 1300 \mathrm{~K}$. Evolutionary properties are given in Table 9 and individual systems are discussed below.

We take comparisons a step further by running additional atmosphere models using a fixed value for effective temperature, gravity, and radius from evolutionary predictions in Table 9. Holding evolutionary properties constant, we ran additional models around the best-fitting cloud properties determined from our grid fits in Section 5 . The goal was to determine if our cloudy atmosphere models could fit data well and remain consistent with substellar evolutionary model predictions.

Because we know the masses for these binary systems, distinguishing between different atmospheric fits becomes more reliable. We are able to eliminate model fits that may represent the data well yet result in implied masses that deviate from empirical observations. Implied mass is calculated from surface gravity and radius for a given atmosphere model. All model fits for individual systems are discussed below. 


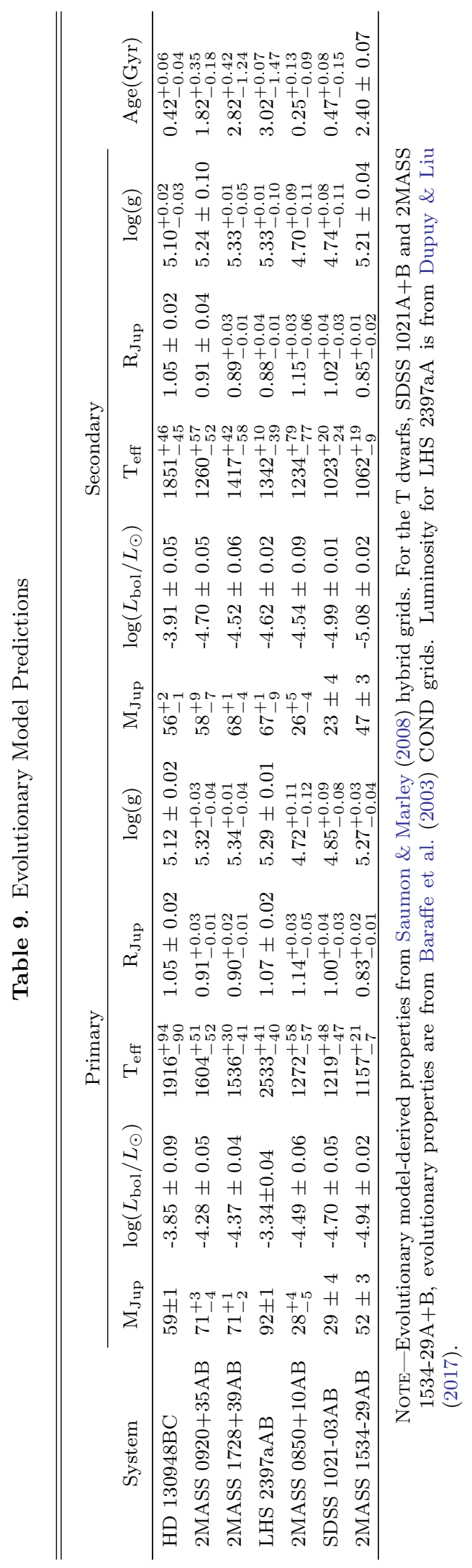




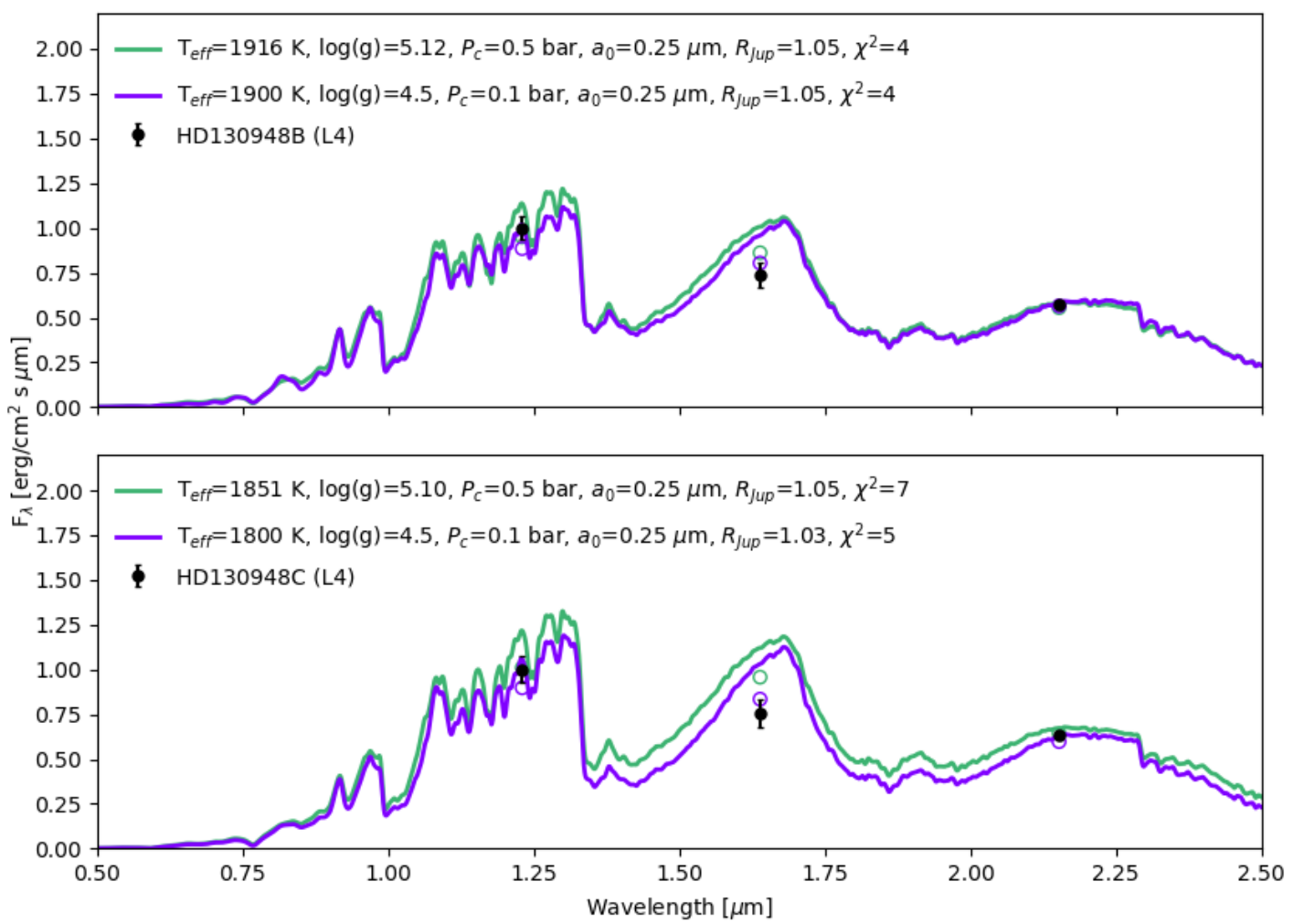

Figure 6. New evolutionary fit atmosphere models (green) compared to weighted mean grid fits (purple) for HD 130948B (top) and HD 130948C (bottom). Surface gravity is in $\mathrm{cm} s^{-2}$.

\section{1. $H D 130948 B+C$}

The best grid fit for the HD 130948B+C system does not match evolutionary-derived properties. This is not surprising because previous work has shown discrepancies between atmosphere grids and evolutionary properties $( \pm$ $250 \mathrm{~K}$ ) when only $J, H$, and $K$ photometry are used (Dupuy et al. 2009). Barman et al. (2011) and other groups find similar issues for directly imaged planets. Additionally, limited SED coverage for this system leads to several well-fit models given grid fits are formally under-constrained. However, by fixing effective temperature, gravity, and radius using evolutionary-derived values we can fit for two cloud parameters to arrive at a more reliable solution that is formally allowed.

In Figure 6 we show our previous weighted mean grid fit compared to our new evolutionary fit with fixed $T_{\text {eff }}, \log (\mathrm{g})$, and $R_{\text {Jup }}$. A good fit to the data can be achieved for HD 130948B with a higher cloud and $a_{0}=0.25 \mu \mathrm{m}$ grain size. The fits are nearly identical, but the grid fit with lower surface gravity is inconsistent with measured mass compared to the higher surface gravity model ( $M_{\mathrm{Jup}}=14.08$ and 58.68 , respectively). HD $130948 \mathrm{C}$ is slightly cooler than the B component yet fit well with the same type of cloud and grain size. Again, the lower surface gravity model can be ruled out by implied mass $\left(M_{\mathrm{Jup}}=13.54\right)$. The new evolutionary fits presented here for both objects are consistent with recent atmospheric properties determined by Briesemeister et al. (2019) which included both $J, H$, and $K$ photometry with additional ALES L-band spectra from 2.9-4.1 $\mu \mathrm{m}$. Our updated fits provide additional constraints on the cloud properties of early-to-mid L type dwarfs.

\subsection{MASS $0850+10 A B$}

We show our new evolutionary fits compared to our previous best-fitting grid model in Figure 7 for 2MASS 0850+10A and B. The initial grid fits significantly over-predicted temperature $(\approx 250-500 \mathrm{~K})$ and gravity $(\approx 0.75$ dex $)$ while underpredicting radius compared to evolutionary predictions. Weighted mean parameters were consistent with evolutionary 
predictions for the secondary component but not for the primary (Table 8 ). Table 9 shows evolutionary predictions for this system have the lowest values of gravity $(\log (\mathrm{g}) \leq 4.75)$ for all the $\mathrm{L}$ dwarfs studied in this sample.

2MASS $0850+10 \mathrm{~A}$ is fit well when bulk properties are fixed to evolutionary values and clouds include a $1 \mu \mathrm{m}$ mean grain size. The warmer grid-based model $\left(T_{\text {eff }}=1800 \mathrm{~K}\right)$ can be ruled out by the larger implied mass $\left(M_{\mathrm{Jup}}=40.04\right)$ and non-physical radius $\left(0.56 R_{\mathrm{Jup}}\right)$ inconsistent with evolutionary models. Figure 7 shows how the grid fit for $2 \mathrm{MASS}$ $0850+10 \mathrm{~B}$ compares to a cooler model with fixed evolutionary parameters. We can rule out the warmer $1500 \mathrm{~K}$ model because the implied mass $\left(69.91 M_{\mathrm{Jup}}\right)$ is higher than the total measured mass of the entire system $\left(M_{\text {tot }}=54 \pm 8\right.$ $\left.M_{\mathrm{Jup}}\right)$. For the $T_{\text {eff }}=1234 \mathrm{~K}$ model, the fit can be improved at $J$ band if the grain size is increased to $1 \mu \mathrm{m}$, but this results in a poor fit to HST photometry near 0.75-1 $\mu \mathrm{m}$. A deeper cloud can also improve the $J$-band fit but reddens the $H$ and $K$ bands.

We believed the warmer, initial grid fit for this system, notably the primary, may have been caused by the spacing of our grid since evolutionary-predicted gravities for 2MASS $0850+10 \mathrm{AB}$ are near the edge of a boundary. To test this hypothesis and determine if the issue was indeed lack of sufficient grid coverage, we extended the segment of our grid that begins at $\log (\mathrm{g})=4.75$ down to $\log (\mathrm{g})=4.5$, including the same range of temperatures and cloud properties given in row two of Table 7 . We then recalculated the grid fits for both A and B components. The best grid fit for 2MASS 0850+10A did not change with the addition of new lower gravity grid models. The weighted mean properties remained nearly identical to our previous findings and inconsistent with evolutionary predictions. The updated best grid fit for 2MASS $0850+10 \mathrm{~B}$ resulted in an unusually cool model with a non-physical radius. Weighted mean parameters were again consistent with predictions from evolutionary models within the uncertainties, but the average gravity was still higher than expected $\left(\log (\mathrm{g})=5.25_{+0.25}^{-0.77}\right)$.

Previous work has suggested 2MASS 0850+10A may be an unresolved binary. Burgasser et al. (2011) pointed out the object had unusually bright $J$ and $K$-band absolute magnitudes for a late L dwarf. Dupuy \& Liu (2012, 2017) later determined with an updated system distance absolute magnitudes were similar to other L5-L7 dwarfs. Our HST photometry shows large differences in component brightnesses across multiple bands $(\Delta \mathrm{F} 625 \mathrm{~W}=1.31 \mathrm{dex}, \Delta \mathrm{F} 775 \mathrm{~W}$ $=1.16 \mathrm{dex}, \Delta \mathrm{F} 814 \mathrm{~W}=1.47 \mathrm{dex}$, and $\Delta \mathrm{F} 850 \mathrm{LP}=0.86 \mathrm{dex}$ ) hinting 2MASS 0850+10A might actually be composed of more than one object after all. The small total system mass of $54 \pm 8 M_{\text {Jup }}$ implies 2MASS $0850+10$ A would be a pair of objects near the deuterium burning limit $\left(\approx 13.5 M_{\mathrm{Jup}}\right)$ with lower gravity $\left(\log (\mathrm{g})=4.3, R_{\mathrm{Jup}}=1.3\right)$ assuming the primary is an equal mass binary, and the B component is a more massive single object with higher gravity $(\log (\mathrm{g})=4.70$, $R_{\text {Jup }}=1.15$ ). The effective temperature in substellar evolutionary models can be quite flat for objects near $20 M_{\text {Jup }}$ at these ages due to clouds, and low-mass objects of similar effective temperatures have been known to look like mid-tolate L dwarfs (Barman et al. 2011). Fitting the A component as a binary would require more specialized atmosphere models.

\subsection{MASS $1728+39 A B$}

The best grid fit for 2MASS $1728+39 \mathrm{~A}$ is consistent with evolutionary predictions in temperature and radius but gravity is over-predicted resulting in an implied mass that is inconsistent with measured masses $\left(M_{\mathrm{Jup}}=115.22\right)$. By fixing $\mathrm{T}_{\text {eff }}, \log (\mathrm{g})$, and $\mathrm{R}_{\mathrm{Jup}}$ to evolutionary predictions from Table 9 and adjusting the depth of the cloud, we are able to produce a good fit to the data shown in Figure 8. The $1536 \mathrm{~K}$ model has an implied mass consistent with measured mass $\left(M_{\mathrm{Jup}}=71.54\right)$.

Fitting 2MASS 1728+39B was not as straightforward as fitting the A component. It was difficult to find a model fit that agreed reasonably well with the data simultaneously in both the F814W and F1042W bands even with fixed $\mathrm{T}_{\text {eff }}$, $\log (\mathrm{g})$, and $\mathrm{R}_{\mathrm{Jup}}$ to evolutionary predictions. The $\mathrm{F} 814 \mathrm{~W}$ data point was fit best by models with deeper clouds and $0.25 \mu \mathrm{m}$ grains whereas the $\mathrm{F} 1042 \mathrm{~W}$ point preferred models with higher clouds and $\geq 1 \mu \mathrm{m}$ grains. Figure 8 shows evolutionary model fits with both cloud preferences compared to our initial grid fit. The lower gravity $1400 \mathrm{~K}$ model can be excluded because its implied mass of $M_{\mathrm{Jup}}=31.98$ is less than half of the measured mass. For the remainder of this paper, we use the model fit that excludes the $\mathrm{F} 814 \mathrm{~W}$ photometric point as our preferred fit because it is more consistent with the majority of the photometric bands.

2MASS $1728+39 \mathrm{AB}$ is a flux reversal binary system, which may explain some of the difficulty finding an atmosphere model that fit in all bands. Several binary systems have been discovered with a secondary component brighter than the primary component in the 1.0-1.3 $\mu \mathrm{m}$ range (Gelino et al. 2014). In this system, the B component is brighter in the F1042W band $\left(\Delta_{A-B}=0.25\right.$ dex) but not in the $J$ or F814W bands. Looper et al. (2008a) explain the brightening could be the result of a cryptobinary, but it is often an intrinsic property of the object due to weather, unusual cloud 


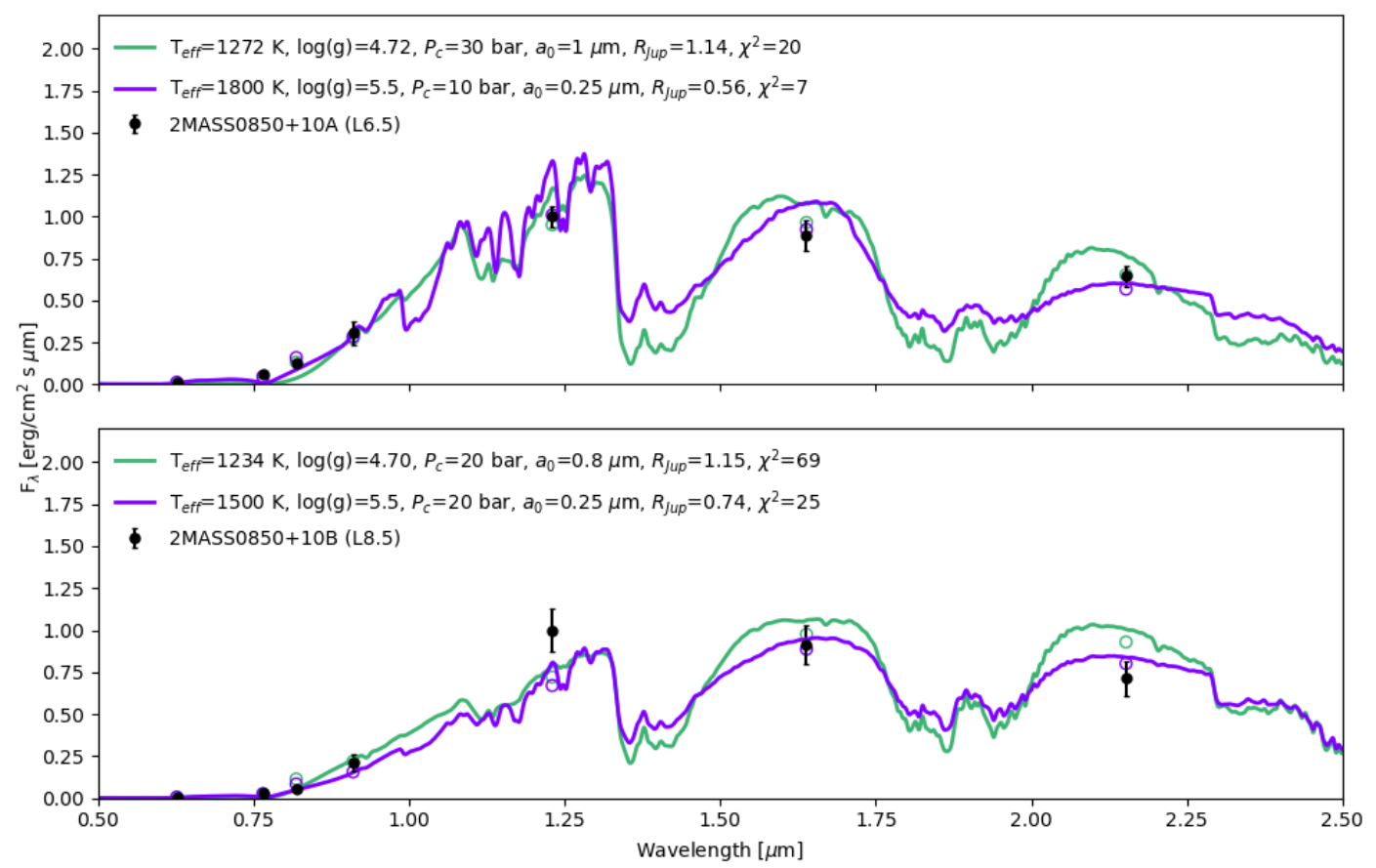

Figure 7. New evolutionary fit atmosphere models (green) compared to best grid fits (purple) for $2 \mathrm{MASS} 0850+10 \mathrm{~A}$ (top) and 2MASS $0850+10 \mathrm{~B}$ (bottom). Surface gravity is in $\mathrm{cm} s^{-2}$.

properties, or changes in surface gravity. Surface gravity is unlikely to be the culprit because widely varying gravities requires different ages not expected for coeval binaries (Gelino et al. 2014). Our model fits suggest it may be due to differences in cloud location and particle sizes.

\subsection{MASS $0920+35 A B$}

Dupuy \& Liu (2017) suggest 2MASS $0920+35$ AB is a triple brown dwarf system given the large total mass of the system $\left(187 \pm 11 M_{\mathrm{Jup}}\right)$ with over half of the total system mass belonging to the fainter secondary $\left(116_{-8}^{+7} M_{\mathrm{Jup}}\right)$. In order to obtain evolutionary predictions for the A component of this system, we follow the same approach as Dupuy \& Liu (2017) and assume 2MASS 0920+35B is composed of two equal-mass, equal-luminosity components. These predictions are given in Table 9.

Figure 9 compares our best grid-fit model of 2MASS 0920+35A from Section 5 to a new model with fixed $\mathrm{T}_{\text {eff }}, \log (\mathrm{g})$, and $\mathrm{R}_{\mathrm{Jup}}$ from evolutionary predictions. We are able to produce a good fit to the data and remain consistent with evolutionary predictions if the B component is indeed a binary with two equal-mass, equal-luminosity components. We derive a younger age of the system at $1.82_{-0.18}^{+0.35}$ Gyr using SM08 evolutionary models, but our results are consistent within the uncertainties to the system age from Dupuy \& Liu (2017) of $2.3_{-0.4}^{+0.3}$ Gyr.

\section{5. $L H S 2397 a A B$}

We only studied the L dwarf companion in this system because the A component is a low-mass star and outside the temperature range of our grid fitting ( $\geq 2000 \mathrm{~K}$ ). Although the best grid-fitting model for LHS 2397aB was consistent in temperature and radius to evolutionary properties, the lower gravity resulted in an implied mass that was too small $\left(M_{\mathrm{Jup}}=20.49\right)$. By fixing the bulk atmospheric properties of the object to evolutionary-derived properties from Table 9, we are able to get a good fit to the data with a new model show in Figure 10 that has an implied mass consistent with observations $\left(M_{\mathrm{Jup}}=66.84\right)$.

\subsection{SDSS 1021-03AB}

It became apparent our cloudy atmosphere grids did not sample enough of the parameter space in cloud pressure and grain size to accommodate early-to-mid $\mathrm{T}$ dwarfs, particularly at lower values of surface gravity near $\log (\mathrm{g})=4.75$. 


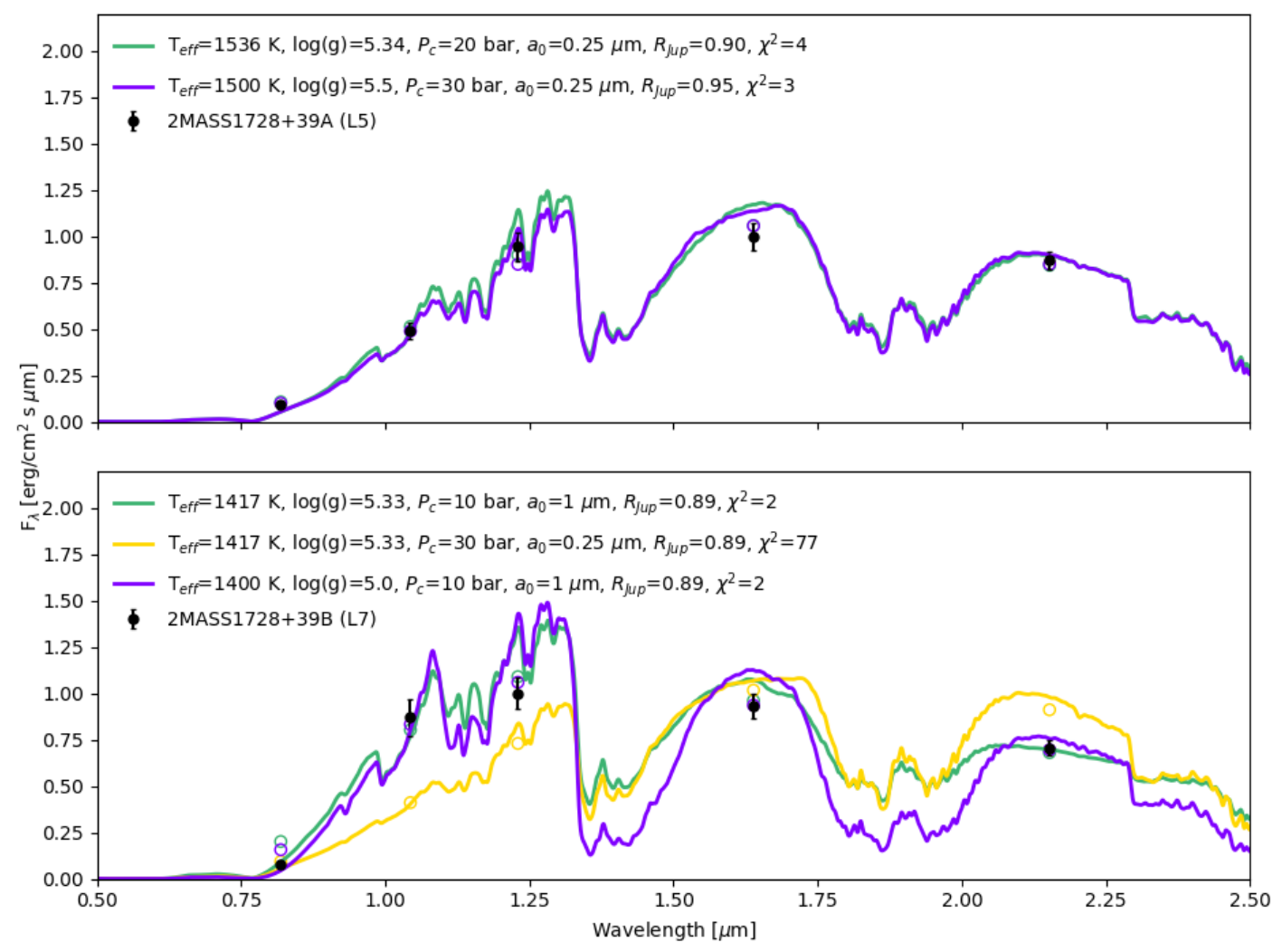

Figure 8. New evolutionary fit atmosphere models (green) compared to best grid fits (purple) for 2MASS 1728+39A (top) and 2MASS 1728+39B (bottom). Two fits for 2MASS 1728+39B are provided: one excluding the F814W photometric point (green) and one including it (gold). Surface gravity is in $\mathrm{cm} \mathrm{s}^{-2}$.

$K$-band flux is sensitive to surface gravity (Saumon et al. 2012), and the cooler models in our grid more appropriate for $\mathrm{T}$ dwarfs predicted an overly red $H-K$ color. We were able to obtain improved fits more appropriate for the SDSS 1021-03AB system by testing models outside the grid with larger grain sizes and deeper clouds.

The cloudy model fits presented here help to provide an upper limit to the location of clouds in $\mathrm{T}$ dwarf atmospheres, which are traditionally fit with cloud-free models (Baraffe et al. 2003). Figure 11 provides a comparison of the best grid models to new evolutionary-based models. The initial grid fits to the system have lower values of $\chi^{2}$ but significantly over-predicted temperature $(\geq 500 \mathrm{~K})$ and under-predicted radius $\left(\leq 0.50 R_{\mathrm{Jup}}\right)$. Although the $J$-band flux is underpredicted by our evolutionary fit for SDSS 1021-03B, this model is still the preferred model based on mass and radius. If we allow the radius to vary, the fit at $J$-band can be improved using a model with a deeper cloud and larger grain size $\left(P_{c}=100\right.$ bar, $\left.a_{0}=5 \mu \mathrm{m}\right)$ with a radius of $1.25 R_{\mathrm{Jup}}$; however, this results in a $35 M_{\text {Jup }}$ mass inconsistent with observations of $23 \pm 4$.

\subsection{MASS 1534-29AB}

Similar to the SDSS 1021-03AB system, the mid-T dwarfs in the 2MASS 1534-29AB system were not fit well by our cloudy atmosphere grids. Grid fits resulted in significantly over-predicted effective temperatures $(\geq 500 \mathrm{~K})$ and small, non-physical radii ( $\leq 0.40 R_{\mathrm{Jup}}$ ) for both the A and B components.

Using cloud parameters informed from our evolutionary fits to the SDSS 1021-03AB system, we were able to test additional models and find more realistic fits for 2MASS 1534-29AB shown in Figure 12. The fits are greatly improved over the original grid-based models and help to provide upper limits to the cloud over cloud-free models.

\section{CLOUD PROPERTIES}




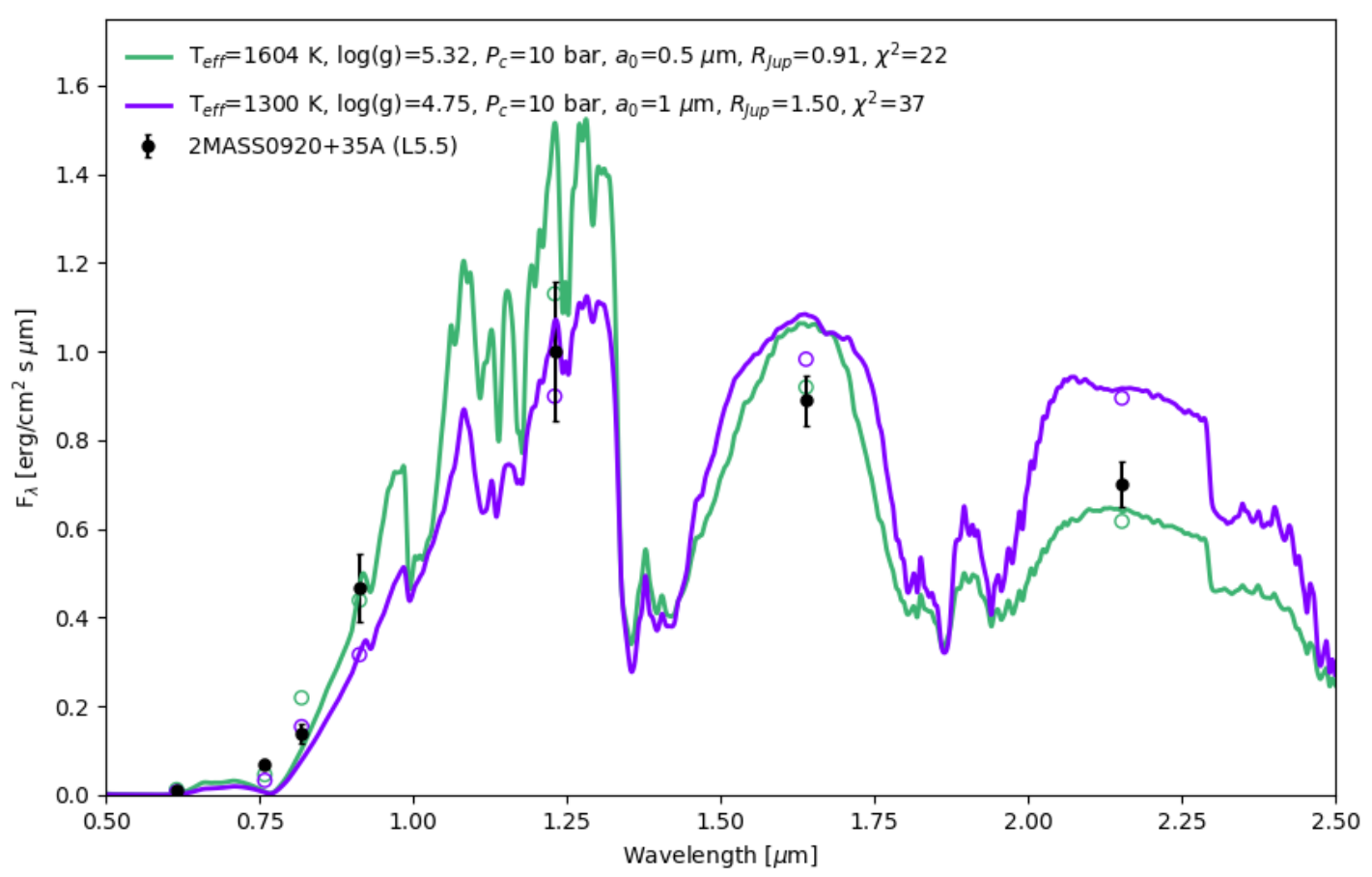

Figure 9. New evolutionary fit atmosphere model (green) compared to best grid fit (purple) for 2MASS 0920+35A. Surface gravity is in $s^{-2}$.

Here we discuss detailed cloud properties from our best-fitting atmosphere models. For the remainder of the paper, we consider the best fits those constrained by evolutionary models in the previous section. Model parameters are summarized in Table 10. We also include the location of the photosphere, cloud location (top, base), cloud thickness, and peak gas-to-dust ratio in the table for reference. We adopt the approximate location of the spectrum-forming region (photosphere) where the atmospheric temperature equals the effective temperature. This location is also very close to where the Rosseland mean optical depth is $\approx 1$.

The model cloud is composed of multiple layers of thermochemically permissible condensate species composed mostly of Fe and Mg-Si grains. Cloud opacity is determined from a heterogeneous mixture of these grains along with a number of minor contributors for which opacities are available rather than using a single grain type as representative of all cloud particles. We report the most abundant condensate species for each object in Table 11. While the table is representative of the composition at the cloud top layer, a gradient of condensates is present within the cloud.

Figure 13 illustrates how the cloud properties change in our sample for $\mathrm{L}$ to mid T-type dwarfs for $\mathrm{T}_{\text {eff }} \approx 1900-1000$ K. Cloud formation occurs higher in the atmosphere and shifts to deeper regions as effective temperature decreases. The peak dust-to-gas ratio shifts to deeper regions within the cloud for cooler objects as well. This trend emerges into two distinct clusters in the plot-one containing eight objects $(\approx$ L4-T5) and one containing four objects $(\approx$ L6-T5)signifying a similar trend for high-gravity and low-gravity objects. It is worth noting all objects in our sample near $T_{\text {eff }}$ $\leq 1400 \mathrm{~K}$ have detached convection zones from the radiative-convective boundary with a convective flux greater than $50 \%$ of the total flux. Isolated convection zones can emerge as temperatures decrease. These detached convection zones are a result of localized changes to the temperature gradient caused by the cloud opacity. Similar zones, produced for the same reason, were discussed by Burrows et al. (2006). In addition to the effective temperature and gravity, the location and vertical extent of these zones are sensitive to the detailed cloud properties (e.g., composition, particle size, and cloud morphology) that determine a cloud's contribution to the total opacity.

Sub-micron grains $(0.25-0.50 \mu \mathrm{m})$ were the most appropriate for L4-L5.5 spectral types with temperatures of $\approx 1900$ $1500 \mathrm{~K}$ and high surface gravities $(\log (\mathrm{g}) \geq 5.0)$ and one lower gravity $(\log (\mathrm{g}) \leq 5.0)$ L8.5 dwarf. Previous work found comparable grain sizes for L dwarfs using a single grain type such as 0.4-0.6 $\mu \mathrm{m}$ for corundum and enstatite grains (Marocco et al. 2014), 0.15-0.3 $\mu \mathrm{m}$ for iron grains (Marocco et al. 2014), and a mean grain size of 0.15-0.35 $\mu \mathrm{m}$ 


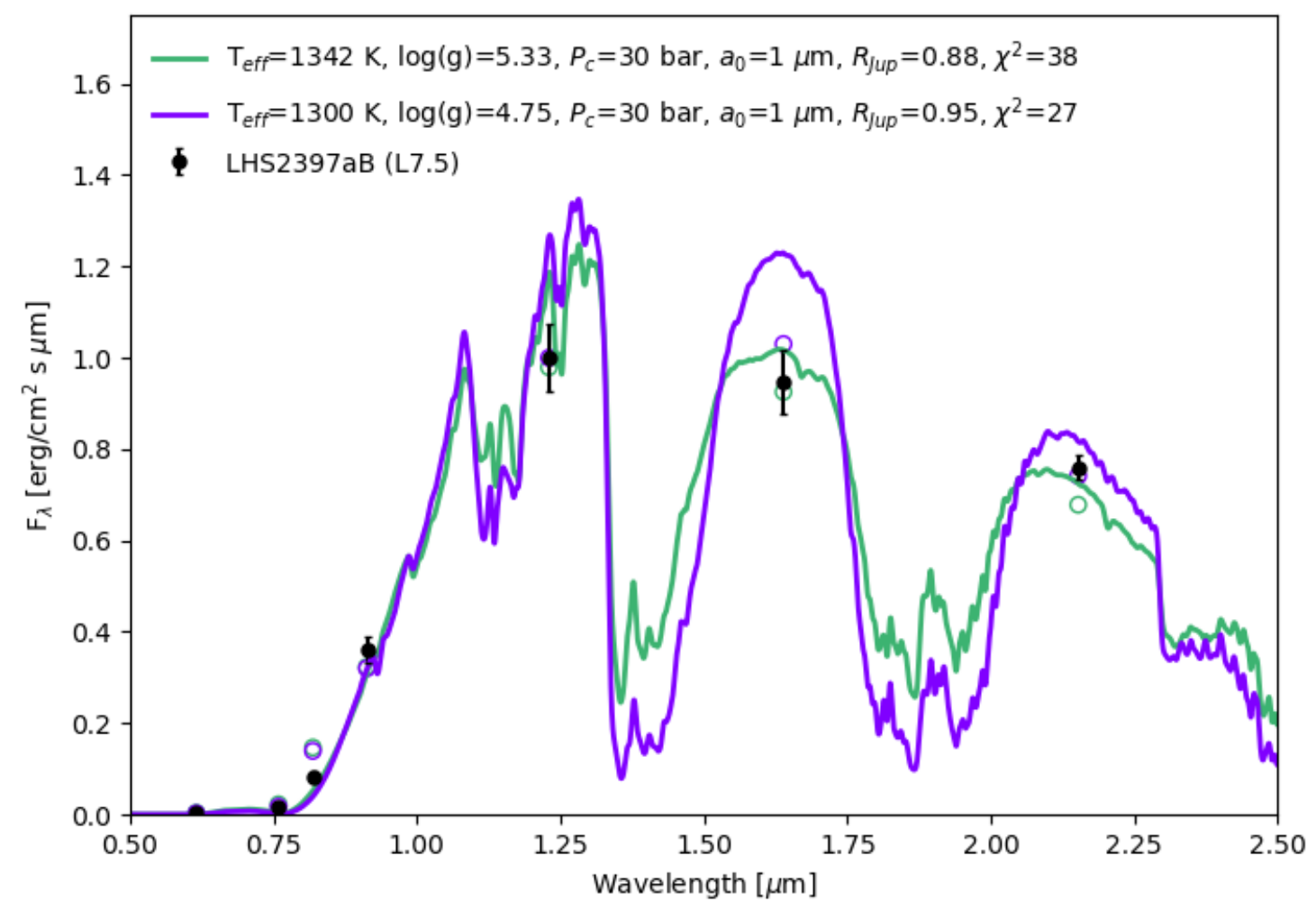

Figure 10. New evolutionary fit atmosphere model (green) compared to the best grid fit (purple) for LHS 2397aB. Surface gravity is in $\mathrm{cm} s^{-2}$.

using forsterite grains (Hiranaka et al. 2016). Rotational modulations have also suggested hazes present in L dwarf atmospheres have characteristic grain sizes of $\approx 0.28-0.4 \mu \mathrm{m}$ (Lew et al. 2016).

Larger grain sizes $(\geq 1 \mu \mathrm{m})$ were required for most spectral types later than L6.5-L7 with effective temperatures near $1400 \mathrm{~K}$ and cooler. The coolest and latest type objects in our sample required the largest grain sizes $(2-5 \mu \mathrm{m})$. Similarly, Zhou et al. (2018) found characteristic condensate particle sizes grew for later L types ( $\geq$ L8) with larger mean grain sizes required to match observations $\left(\mathrm{a}_{0} \geq 1.0 \mu \mathrm{m}\right)$.

We compare our modelled objects to the cooling and color evolution of brown dwarfs across the L/T transition using Saumon \& Marley (2008) hybrid evolution models in Figure 14. Objects redder than the track were best represented by the smallest grain sizes, whereas objects bluer than the track where those that required the largest grain sizes. Reddened L dwarfs in the L5-L7.5 range can be an important indicator of youth in brown dwarfs, or the redder colors can be indicative of excess dust and clouds in the photosphere (Kirkpatrick 2005; Filippazzo et al. 2015; Faherty et al. 2012). The reddest object in our sample, 2MASS $1728+39 \mathrm{~A}$, is a mid-L dwarf slightly redder than average $\left(J-K_{s}=\right.$ 2.07), which we believe is due to excess dust and a small cloud particle size.

\section{SUMMARY AND CONCLUSION}

Discrepancies between atmosphere and evolutionary model predictions play a significant role as different stages of condensate cloud evolution greatly influences the overall spectral shape. Previous grid comparisons have led to disagreements between atmosphere models and evolutionary predictions for substellar objects of known mass (e.g., Chabrier \& Baraffe 2000; Dupuy et al. 2014). We are able to produce atmosphere models that match evolutionary predictions for a sample of brown dwarfs $(\approx$ L4-T5) by allowing enough flexibility within the cloud properties.

Determining the bulk properties (e.g., effective temperature and gravity) of stellar and substellar mass objects is an important step along the way to making inferences about more specific properties, such as metallicity, or the relative abundances of key elements. These bulk properties are frequently estimated by comparing photometric or spectroscopic observations to model atmosphere predictions. For many objects such comparisons yield properties consistent with 


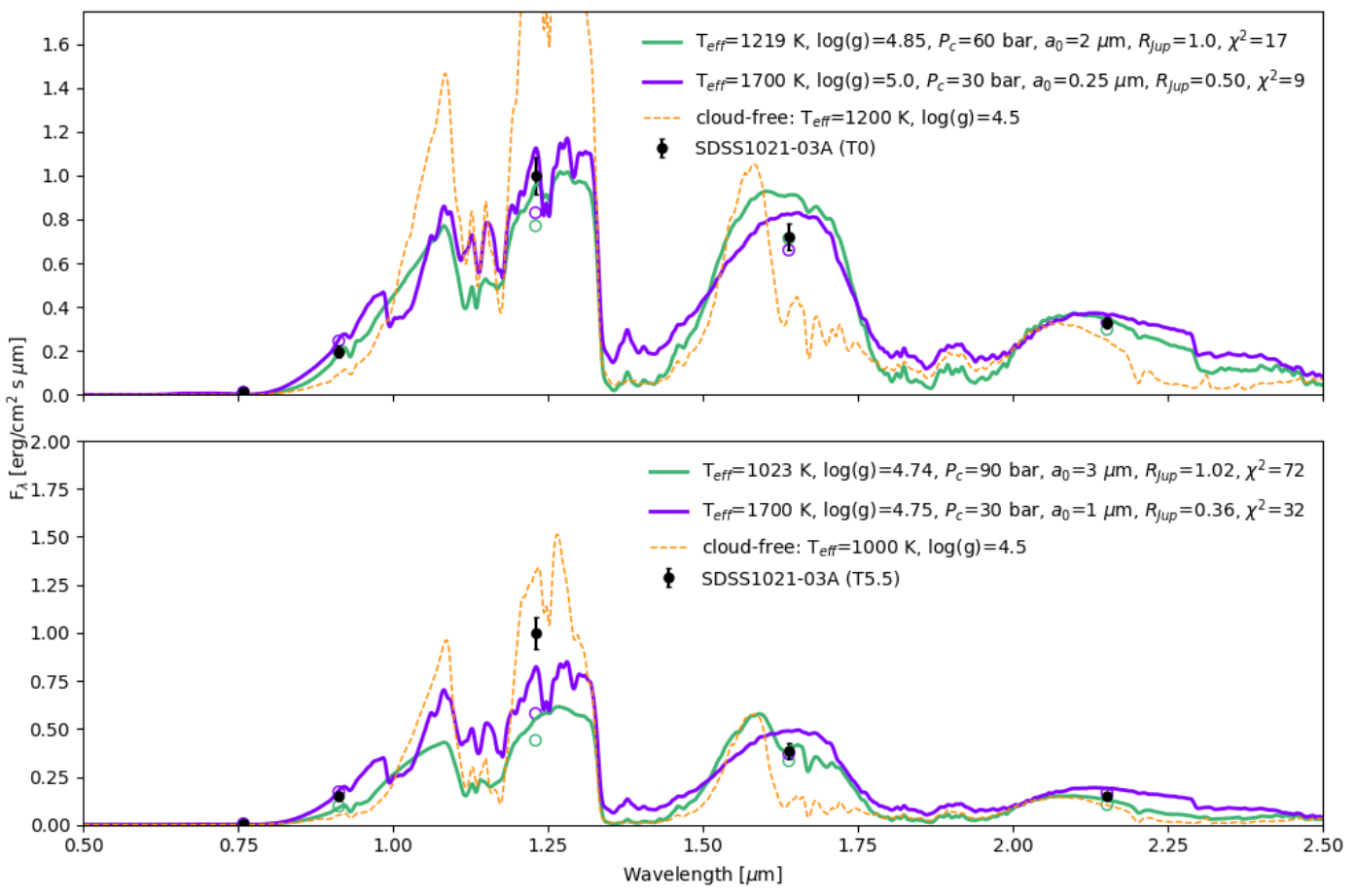

Figure 11. New evolutionary fit atmosphere models (green) compared to best grid fits (purple) for SDSS 1021-03A (top) and SDSS 1021-03B (bottom). Plotted in orange is a cloud-free model for comparison. Surface gravity is in $\mathrm{cm}^{-2}$.

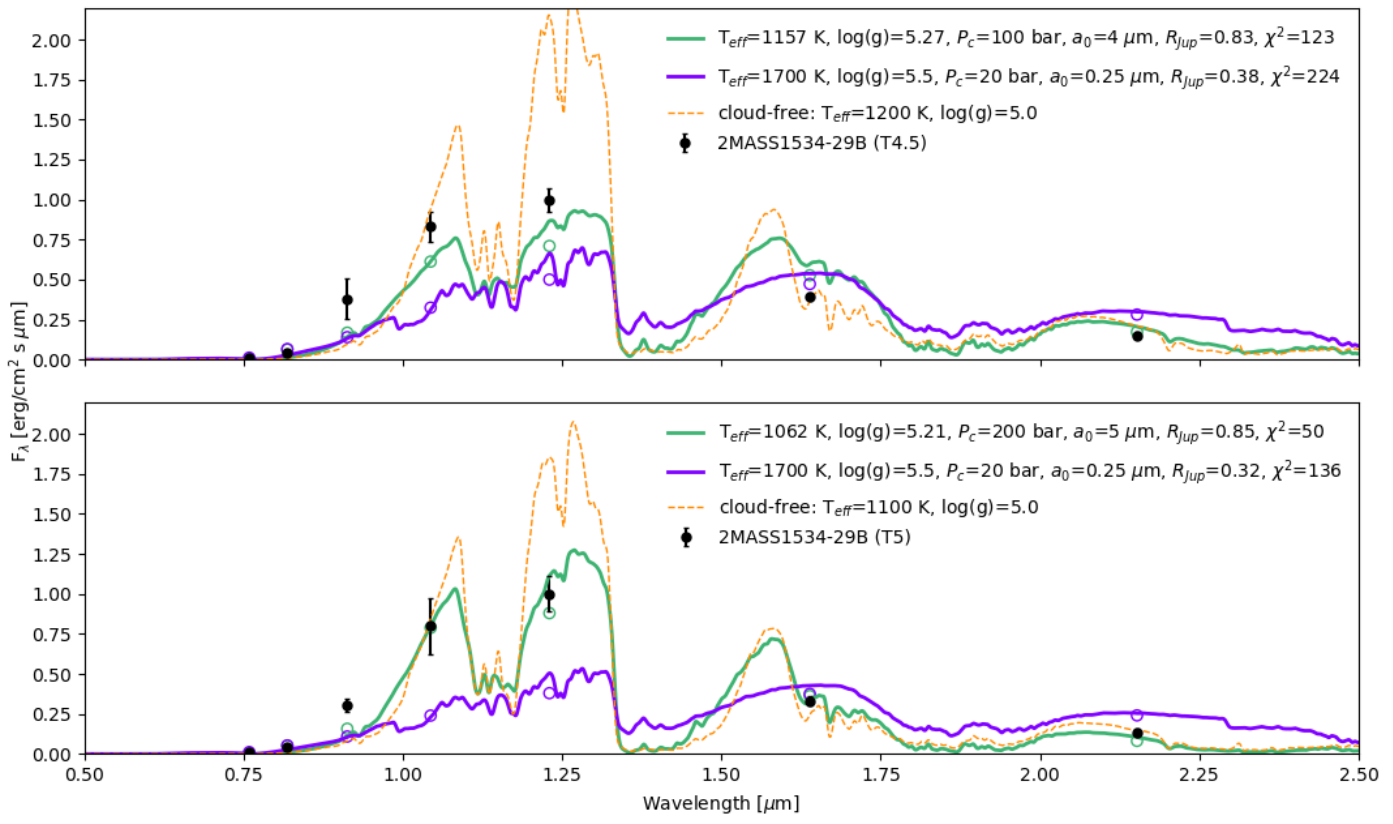

Figure 12. New evolutionary fit atmosphere models (green) compared to best grid fits (purple) for 2MASS 1534-29A (top) and 2MASS 1534-29B (bottom). Plotted in orange is a cloud-free model for comparison. Surface gravity is in $\mathrm{cm}^{-2}$. 


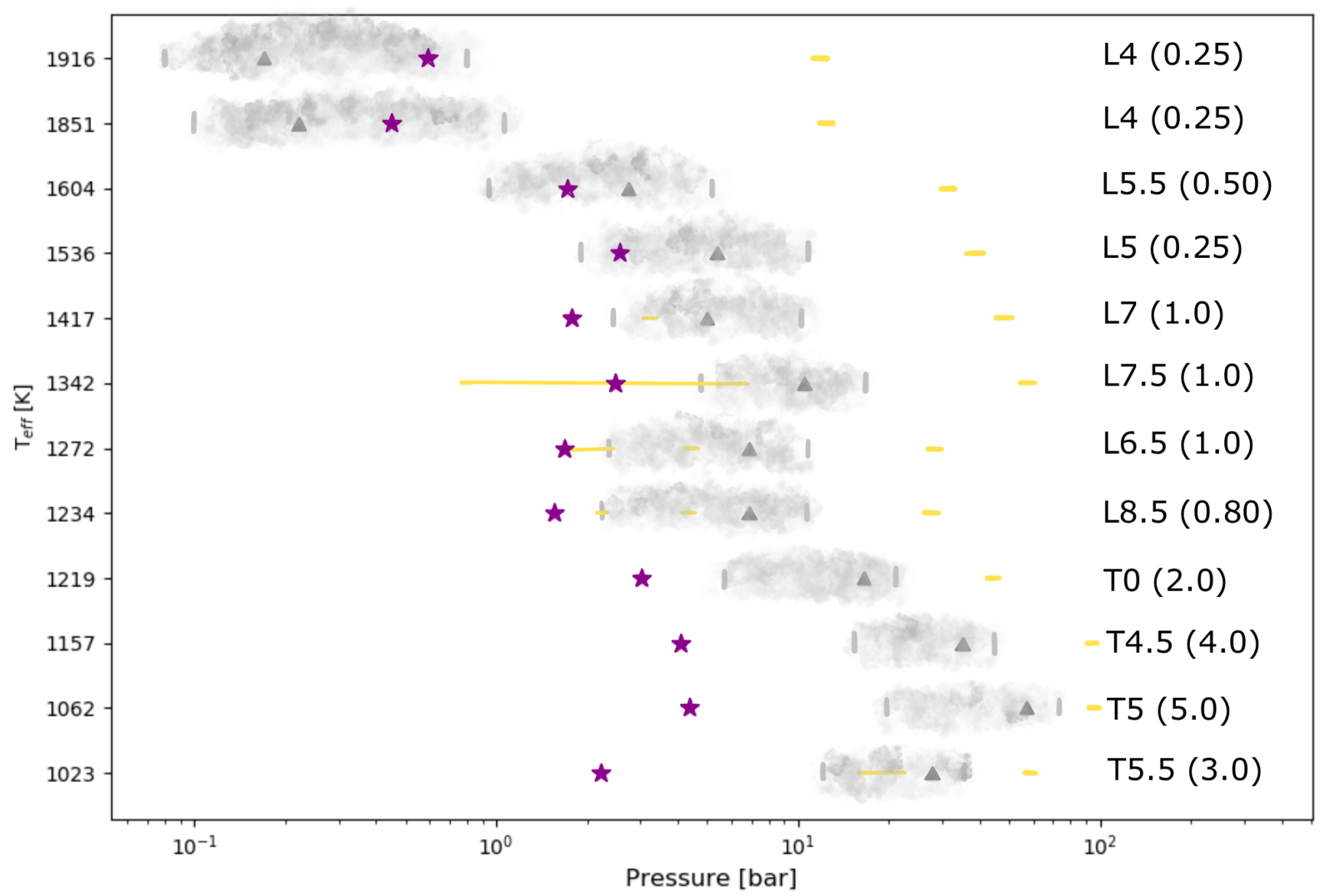

Figure 13. Summary of best-fit evolutionary model cloud progression organized by decreasing effective temperature with the warmest objects at the top. The photosphere for each model atmosphere is represented by a star. The cloud top and cloud base are denoted by light grey lines. The peak dust-to-gas ratio is represented by a grey triangle. Regions with a yellow line are indicative of convection with the right-most dash always indicating the radiative-convective boundary. Yellow regions to the left of the this boundary highlight a detached convection zone $\left(\mathrm{F}_{\text {conv }} / \mathrm{F}_{\text {tot }} \geq 50 \%\right)$. Spectral type and the model's mean grain size in $\mu \mathrm{m}$ is included on the right-hand side of the plot for reference.

those of interior and evolution models that, at moderate to old ages, are considered reliable and less sensitive to model assumptions (Baraffe et al. 2002). Significant inconsistencies, however, can occur whenever condensate cloud formation greatly influences the overall spectral shape. This situation occurs, for example, across the L/T transition (Cushing et al. 2006) and for most young directly imaged companions (Metchev \& Hillenbrand 2006) where models with a range of temperatures, gravities, and cloud properties are capable of matching a single object's near-IR SED equally well (Barman et al. 2011). Such ambiguity can often hinder the study of cloudy objects where mass and age are very uncertain.

In this paper we have studied a set of $\mathrm{L} / \mathrm{T}$ transition $\left(T_{\text {eff }} \approx 1900-1000 \mathrm{~K}\right)$ brown dwarf binaries with measured masses, luminosities, and well-determined ages. For these objects comparisons to evolutionary models yield very precise estimates of the bulk properties. Overall, synthetic spectra from our cloudy atmosphere models matched spatiallyresolved visible to near-IR photometry of each binary component reasonably well. The cloud parameters included in our grids appear to be most appropriate for L4-L8 field dwarfs with effective temperatures between 1900-1300 K and $\log (\mathrm{g}) \geq 5.0$. The grid fits for these objects were the most consistent with our evolutionary-constrained atmosphere models. 
Table 10. Summary of Evolutionary Constrained Cloud Properties

\begin{tabular}{lcccccccccc}
\hline \hline \multicolumn{1}{c}{ Object } & $\mathrm{T}_{\text {eff }}$ & $\log (\mathrm{g})$ & $\mathrm{a}_{0}$ & $\mathrm{P}_{\mathrm{c}}$ & Photosphere & Cloud Base & Cloud Top & Peak Dust & $\mathrm{H}_{p}$ & SpT \\
\hline HD 130948B & 1916 & 5.12 & 0.25 & 0.5 & 0.59 & 0.80 & 0.08 & 0.17 & 1.93 & $\mathrm{~L} 4 \pm 1$ \\
HD 130948C & 1851 & 5.10 & 0.25 & 0.5 & 0.45 & 1.07 & 0.10 & 0.22 & 2.58 & $\mathrm{~L} 4 \pm 1$ \\
2MASS 0920+35A & 1604 & 5.32 & 0.50 & 10 & 1.72 & 5.21 & 0.94 & 2.74 & 2.14 & $\mathrm{~L} 5.5 \pm 1$ \\
2MASS 1728+39A & 1536 & 5.34 & 0.25 & 20 & 2.57 & 10.8 & 1.89 & 5.37 & 2.16 & $\mathrm{~L} 5 \pm 1$ \\
2MASS 1728+39B & 1417 & 5.33 & 1.0 & 10 & 1.78 & 10.2 & 2.45 & 5.00 & 1.90 & $\mathrm{~L} 7 \pm 1$ \\
LHS2397aB & 1342 & 5.33 & 1.0 & 30 & 2.49 & 16.7 & 4.79 & 10.5 & 1.53 & $\mathrm{~L} 7.5 \pm 1$ \\
2MASS 0850+10A & 1272 & 4.72 & 1.0 & 30 & 1.68 & 10.8 & 2.37 & 6.90 & 1.84 & $\mathrm{~L} 6.5 \pm 1$ \\
2MASS 0850+10B & 1234 & 4.70 & 0.8 & 20 & 1.56 & 10.7 & 2.24 & 6.84 & 1.90 & $\mathrm{~L} 8.5 \pm 1$ \\
SDSS 1021-03A & 1219 & 4.85 & 2.0 & 60 & 3.04 & 21.0 & 5.67 & 16.5 & 1.57 & $\mathrm{~T} 0 \pm 1$ \\
2MASS 1534-29A & 1157 & 5.27 & 4.0 & 100 & 4.06 & 44.6 & 15.4 & 34.9 & 1.30 & $\mathrm{~T} 4.5 \pm 1$ \\
2MASS 1534-29B & 1062 & 5.21 & 5.0 & 200 & 4.35 & 73.6 & 19.6 & 57.2 & 1.48 & $\mathrm{~T} 5 \pm 1$ \\
SDSS 1021-03B & 1023 & 4.74 & 3.0 & 90 & 2.21 & 35.4 & 12.1 & 27.8 & 1.26 & $\mathrm{~T} 5.5 \pm 1$ \\
\hline
\end{tabular}

Note-Detailed model cloud properties for best evolutionary-derived atmosphere model fits from Section 6. Cloud properties are given in bars. $\mathrm{H}_{p}$ is the cloud thickness in pressure scale height. Spectral types listed are from Dupuy \& Liu (2017).

Table 11. Most Abundant Condensates in Cloud Top Layer

\begin{tabular}{|c|c|}
\hline Object & Condensates \\
\hline HD 130948B & $\mathrm{Fe}(48 \%)-\mathrm{MgSiO}_{3}, \mathrm{Mg}_{2} \mathrm{SiO}_{4}, \mathrm{MgO}, \mathrm{SiO}_{2}(52 \%)$ \\
\hline HD $130948 \mathrm{C}$ & $\mathrm{Fe}(49 \%)-\mathrm{MgSiO}_{3}, \mathrm{MgO}, \mathrm{Mg}_{2} \mathrm{SiO}_{4}, \mathrm{SiO}_{2}(51 \%)$ \\
\hline 2MASS $0920+35 \mathrm{~A}$ & $\mathrm{Fe}(48 \%)-\mathrm{MgSiO}_{3}, \mathrm{Mg}_{2} \mathrm{SiO}_{4}, \mathrm{MgO}, \mathrm{SiO}_{2}(52 \%)$ \\
\hline 2MASS $1728+39 \mathrm{~A}$ & $\mathrm{Fe}(49 \%)-\mathrm{MgSiO}_{3}, \mathrm{MgO}, \mathrm{Mg}_{2} \mathrm{SiO}_{4}, \mathrm{SiO}_{2}(51 \%)$ \\
\hline 2MASS $1728+39 B$ & $\mathrm{Fe}(50 \%)-\mathrm{MgO}, \mathrm{SiO}_{2}, \mathrm{Mg}_{2} \mathrm{SiO}_{4}, \mathrm{MgSiO}_{3}, \mathrm{MgAl}_{2} \mathrm{O}_{4}(50 \%)$ \\
\hline LHS2397aB & $\mathrm{Fe}(45 \%)-\mathrm{MgSiO}_{3}, \mathrm{MgO}, \mathrm{SiO}_{2}, \mathrm{Mg}_{2} \mathrm{SiO}_{4}(55 \%)$ \\
\hline 2MASS $0850+10 \mathrm{~A}$ & $\mathrm{Fe}(49 \%)-\mathrm{MgSiO}_{3}, \mathrm{MgO}, \mathrm{Mg}_{2} \mathrm{SiO}_{4}, \mathrm{SiO}_{2}(51 \%)$ \\
\hline 2MASS $0850+10 \mathrm{~B}$ & $\mathrm{Fe}(48 \%)-\mathrm{MgSiO}_{3}, \mathrm{Mg}_{2} \mathrm{SiO}_{4}, \mathrm{MgO}, \mathrm{SiO}_{2}(52 \%)$ \\
\hline SDSS 1021-03A & $\mathrm{Fe}(47 \%)-\mathrm{MgSiO}_{3}, \mathrm{MgO}, \mathrm{Mg}_{2} \mathrm{SiO}_{4}, \mathrm{SiO}_{2}(53 \%)$ \\
\hline 2MASS $1534-29 \mathrm{~A}$ & $\mathrm{Fe}(45 \%)-\mathrm{SiO}_{2}, \mathrm{MgO}, \mathrm{Mg}_{2} \mathrm{SiO}_{4}, \mathrm{MgSiO}_{3}, \mathrm{MgAl}_{2} \mathrm{O}_{4}(55 \%)$ \\
\hline 2MASS 1534-29B & $\mathrm{Fe}(46 \%)-\mathrm{SiO}_{2}, \mathrm{MgO}, \mathrm{Mg}_{2} \mathrm{SiO}_{4}, \mathrm{MgSiO}_{3}, \mathrm{MgAl}_{2} \mathrm{O}_{4}(54 \%)$ \\
\hline SDSS 1021-03B & $\mathrm{Fe}(47 \%)-\mathrm{MgSiO}_{3}, \mathrm{MgO}, \mathrm{SiO}_{2}, \mathrm{Mg}_{2} \mathrm{SiO}_{4}(53 \%)$ \\
\hline
\end{tabular}

Note-The most abundant dust species are listed for each object at the cloud top layer. Individual condensates species are in order from the most abundant to the least abundant and represent a combination of both solid and liquid phases. Percentages shown are for total iron grains and $\mathrm{Mg}-\mathrm{Si}$ grains, respectively.

With these atmosphere models we have determined a set of cloud properties across the $\mathrm{L} / \mathrm{T}$ transition. The warmest objects in the sample $(\approx 1900-1500 \mathrm{~K})$ were fit best by particles with mean grain sizes of $0.25-0.50 \mu \mathrm{m}$, whereas objects cooler than $1500 \mathrm{~K}$ required larger mean grain sizes 0.80-5 $\mu \mathrm{m}$. Although the composition at the cloud top remained relatively close to an equal split between $\mathrm{Fe}$ and $\mathrm{Mg}$-Si grains for the majority of objects, the overall location of the cloud top shifted to deeper regions within the atmosphere as objects cooled in effective temperature. Near $1400 \mathrm{~K}$ clouds began to disappear below the photosphere, which agrees with previous findings (Saumon \& Marley 2008; Marley et al. 2010).

There was some disagreement between our grid-based atmosphere models and the evolutionary-constrained atmosphere models for lower-gravity objects $(\log (\mathrm{g}) \leq 5.0)$ and the latest $\mathrm{T}$ dwarfs included in the sample $\left(\mathrm{T} 4.5\right.$-T5.5; $\mathrm{T}_{\text {eff }}$ $\leq 1200 \mathrm{~K})$. Our grid-based models tended to over-predict temperature, gravity, and under-predict radius. In addition to model-grid aspects (e.g., grid spacing and boundaries), unresolved binarity might play a role in some systems. For example 2MASS $0850+10$ A may be a pair of objects near the deuterium burning limit, rather than a single L-type 

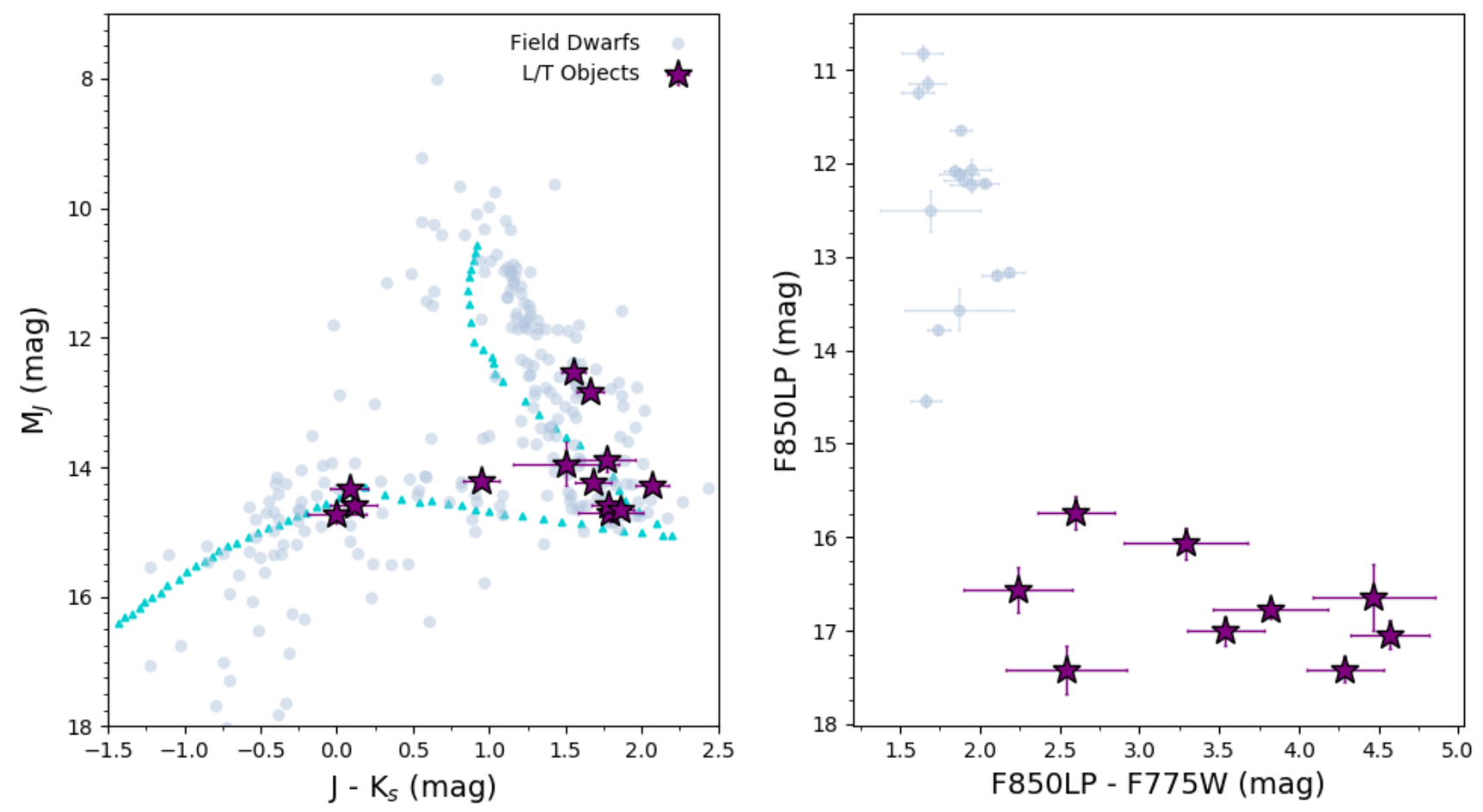

Figure 14. Color-magnitude diagrams in near-infrared and optical colors. Saumon \& Marley (2008) hybrid evolutionary tracks are overplotted in turquoise triangles. Purple stars show the objects focused on in this paper. Filled light blue circles are low-mass field objects from Faherty et al. (2012) (left) and low-mass objects from Konopacky et al. (2010) (left and right). HST observations on right include both WFC3 and ACS filters.

brown dwarf, due to the large difference in brightness between A and B components, low total mass of the system, and mixed results from atmosphere model fitting. Giant exoplanets overlap with the range of effective temperatures of brown dwarfs (Faherty et al. 2016) and such objects (e.g., HR8799c) can resemble mid-L spectral types (Marois et al. 2010; Barman et al. 2011). Binarity is just one possibility and further study, both observationally and modeling, is warranted for this system.

SDSS 1021-03A is the earliest $\mathrm{T}$ dwarf in the sample, and evolutionary-constrained models preferred a deeper cloud $\left(\mathrm{P}_{c}=60\right.$ bar $)$ and larger grain size $(>1 \mu \mathrm{m})$ slightly beyond the parameters in our atmosphere grids. Similarly, the later T4-T5 dwarfs (SDSS 1021-03B, 2MASS 1534-29A, 2MASS 1534-29B) required even deeper clouds $\left(\mathrm{P}_{c}=90-200\right.$ bar) and the largest grain sizes $(3-5 \mu \mathrm{m})$. Evolutionary-derived fits for these objects suggest our grids should be extended to include a larger range of cloud properties to accommodate early-to-mid $\mathrm{T}$ dwarfs and help constrain the limits of homogeneous cloud models for the coolest objects. We observe that condensate growth becomes more important near late-L types, leading to preferred model fits with larger mean grain sizes for $\mathrm{T}$ dwarfs. Other work has successfully reproduced T dwarf photometry using thin sulfide clouds (Morley et al. 2012) and inhomogeneous cloud cover with low temperature condensates $\left(\mathrm{Na}_{2} \mathrm{~S}, \mathrm{KCl}\right.$ ) (Charnay et al. 2018). Near $\mathrm{T}_{\text {eff }} \approx 1000 \mathrm{~K}$ cloud-free models may be a better fit to our data; however, this is the same regime in low-gravity objects where sulfide clouds appear while iron and silicate clouds are simultaneous disappearing (Morley et al. 2012; Charnay et al. 2018). A more diverse grid for these objects will be beneficial in order to untangle the relationship between condensate growth, cloud composition, and surface gravity.

Rapid color changes across the $\mathrm{L} / \mathrm{T}$ transition have been interpreted as the result of patchy clouds or holes in the cloud deck (Burrows et al. 2003; Ackerman \& Marley 2001; Marley et al. 2010), a sudden collapse of the cloud deck (Tsuji \& Nakajima 2003), or an increase in sedimentation efficiency of clouds (Knapp et al. 2004). By parameterizing the sedimentation efficiency of dust particles to regulate the influence of cloud opacity on the model spectrum, one can reproduce the L/T transition (Saumon \& Marley 2008; Stephens et al. 2009). We are able to reproduce photometric changes across the $\mathrm{L} / \mathrm{T}$ transition in a similar fashion by parameterizing the vertical extent and mean grain size of 
a uniform cloud. However, we cannot rule out the existence of patchy clouds in this sample of objects despite our homogeneous cloudy model fits because the presence of cloud holes is very subtle across the near-infrared part of the spectrum for L/T transition objects (Marley et al. 2010; Apai et al. 2013).

Other groups use a more complex treatment of grain nucleation, growth, evaporation, and/or drift. Work from Helling et al. (2008b,a) resulted in mean cloud particle sizes that increased as a function of atmospheric depth, with small particles $(\approx 0.01 \mu \mathrm{m})$ in high atmospheric layers with a narrow grain size distribution that broadened to larger particle sizes $(\approx 100 \mu \mathrm{m})$ and grain size distributions near the cloud base. Charnay et al. (2018) was able to reproduce the spread of near-IR colors across the L/T transition and those observed in reddened low-gravity objects by computing cloud particle radii estimated from simple microphysics. Our models use a constant grain size distribution with cloud height, but similar to more complex treatment of grains, the peak dust-to-gas ratio sinks to deeper regions within the atmosphere, eventually below observable layers.

It has been hinted at that grain size increases as objects cool across the L/T transition (Zhou et al. 2018; Knapp et al. 2004). Burrows et al. (2006) used models with homogeneous forsterite grains with sizes of 3, 10, 30, and 100 $\mu \mathrm{m}$ and identified that atmospheres with larger particles resulted in stronger $J$-band fluxes near $1400-1500 \mathrm{~K}$ due to the natural deepening of the cloud position in the model atmospheres. We observe something similar in our grid models across a smaller range of particle sizes $(0.25-1 \mu \mathrm{m})$ for our heterogeneous grains. Figure 3 shows $J$-band flux is greater for the largest grain sizes at $1300 \mathrm{~K}$ whereas $J$-band fluxes are similar at $1500 \mathrm{~K}$ regardless of grain size. A physical mechanism responsible for this trend in increasing grain size is not well understood. At cooler effective temperatures, it has been suggested that a larger supply of condensate vapor near the base of the cloud could result in runaway particle growth for cooler objects (Gao et al. 2018).

Photometry has limited sensitivity; therefore, future steps will be to improve atmospheric constraints with resolved spectroscopy. Cloud location, mean grain size, surface gravity, and metallicity impact our understanding substellar atmospheres as a function of temperature. Decoupling the degeneracies between these interwoven features is essential to explain observations of objects, particularly across the $\mathrm{L} / \mathrm{T}$ transition. This work provides valuable insight into the complex evolution of cloud opacity for a range of well-studied brown dwarf binaries. Ideally, the goal is to be able to construct reliable atmosphere models that can account for the drastic color change and physical properties of substellar objects without a reliance on evolutionary models to infer the properties of individual objects. We plan to extend our cloudy grids to both lower and higher cloud top pressures with additional grain sizes to accommodate the earliest and latest spectral types. Additional exploration of cloud properties is warranted for the warmest and coolest objects for binaries where our atmosphere grids lacked coverage.

Future work would greatly benefit from additional observations, especially at the shortest wavelength portion of the SED. Photometric bands near 0.8-1 $\mu \mathrm{m}$ appear important when investigating flux reversal binary systems and provide insight to differing grain size preferences at optical and near-infrared wavelengths. The next generation of telescopes with higher sensitivity and wavelength coverage will be essential in providing high-quality spectra required to fine tune cloud parameters and address lingering inconsistencies, such as broad, continuous wavelength coverage from the James Webb Space Telescope's NIRSpec instrument (0.6-5.3 $\mu \mathrm{m})$. Furthermore, variability has been detected in brown dwarfs at the transition region (Radigan 2014) and will be an important factor for cloud formation and evolution going forward. Rotation-modulated spectral variations will be a key approach toward a more in-depth grasp of the evolving cloud structure in low-mass objects (Apai et al. 2013, 2017). Understanding the relationship between grain size distribution and effective temperature will require a multidimensional approach to grain kinetics and growth connected to convective cloud structure. Atmospheric retrieval results can be compared to those of self-consistent models to better understand the nature of discrepancies (e.g., heterogeneous cloud layers, particle sizes, cloud composition, haze layers). Relatively few brown dwarfs spanning the L/T transition have known masses, and the release of future Gaia data will increase parallax precision by $30 \%$ (Collaboration et al. 2020). Objects with independently constrained properties from dynamical mass and luminosity measurements are the strongest candidates for future model comparisons.

\section{ACKNOWLEDGEMENTS}

L. S. B. is extremely grateful for Kyle Pearson and many helpful discussions of this work. We would also like to thank the anonymous referee for a constructive report. Support for program 11605 was provided by NASA through a grant from the Space Telescope Science Institute, which is operated by the Associations of Universities for Research in Astronomy, incorporated under NASA contract NAS5-26555. This work was also supported by NSF grants 1405505 and 1614492. Material presented in this work is supported by the National Aeronautics and Space Administration under 
Grants/Contracts/Agreements No.NNX17AB63G issued through the Astrophysics Division of the Science Mission Directorate. This research makes use of data products from 2MASS, a joint project of the University of Massachusetts and the Infrared Processing and Analysis Center/California Institute of Technology, funded by the National Aeronautics and Space Administration and the National Science Foundation. Some of the data presented herein were also obtained at the W. M. Keck Observatory, which is operated as a scientific partnership among the California Institute of Technology, the University of California, and the National Aeronautics and Space Administration. The Observatory was made possible by the generous financial support of the W. M. Keck Foundation. The authors also recognize and acknowledge the very significant cultural role and reverence that the summit of Mauna Kea has always had within the indigenous Hawaiian community. We are most fortunate to have the opportunity to conduct observations from this mountain. Additionally, this research has benefited from the SpeX Prism Library, maintained by Adam Burgasser at http://www.browndwarfs.org/spexprism.

Software: TinyTim (Krist et al. 2011), StarFinder (Diolaiti et al. 2000), img2xym (Anderson \& King 2006), PHOENIX (Hauschildt 1993; Hauschildt \& Baron 2006; Baron \& Hauschildt 2007)

\section{REFERENCES}

Ackerman, A. S., \& Marley, M. S. 2001, The Astrophysical Journal, 556, 872, doi: 10.1086/321540

Allard, F. 2013, Proceedings of the International Astronomical Union, 8, 271, doi: $10.1017 / \mathrm{S} 1743921313008545$

Allard, F., Hauschildt, P. H., Alexander, D. R., Tamanai, A., \& Schweitzer, A. 2001, The Astrophysical Journal, 556, 357, doi: 10.1086/321547

Anderson, J., \& King, I. R. 2006, PSFs, Photometry, and Astronomy for the ACS/WFC, Tech. rep., STsCI. http: //adsabs.harvard.edu/cgi-bin/nph-data_query?bibcode= 2006acs..rept....1A\&link_type=ABSTRACT\%5Cnpapers: //fedb1a86-f693-4042-a040-86e952d868c2/Paper/p1757

Apai, D., Radigan, J., Buenzli, E., et al. 2013, Astrophysical Journal, 768, doi: 10.1088/0004-637X/768/2/121

Apai, D., Karalidi, T., Marley, M. S., et al. 2017, Science, 357, 683, doi: 10.1126/science.aam9848

Baraffe, I., Chabrier, G., Allard, F., \& Hauschildt, P. H. 2002, Astronomy and Astrophysics, 382, 563, doi: 10.1051/0004-6361:20011638

Baraffe, I., Chabrier, G., Barman, T. S., Allard, F., \& Hauschildt, P. H. 2003, Astronomy and Astrophysics, 402, 701, doi: 10.1051/0004-6361:20030252

Barman, T. S., Konopacky, Q. M., Macintosh, B., \& Marois, C. 2015, Astrophysical Journal, 804, 1, doi: 10.1088/0004-637X/804/1/61

Barman, T. S., MacIntosh, B., Konopacky, Q. M., \& Marois, C. 2011, Astrophysical Journal Letters, 735, 2, doi: 10.1088/2041-8205/735/2/L39

Baron, E., \& Hauschildt, P. H. 2007, Astronomy and Astrophysics, 468, 255, doi: 10.1051/0004-6361:20066755

Briesemeister, Z. W., Skemer, A. J., Stone, J. M., et al. 2019, The Astronomical Journal, 157, 244, doi: 10.3847/1538-3881/ab1901
Burgasser, A., Liu, M. C., Ireland, M. J., Cruz, K. L., \& Dupuy, T. J. 2008a, Astrophysical Journal, 681, 579

Burgasser, A. J. 2014, in Astronomical Society of India Conference Series, Vol. 11, Astronomical Society of India Conference Series, 7-16.

https://arxiv.org/abs/1406.4887

Burgasser, A. J., Bardalez-Gagliuffi, D. C., \& Gizis, J. E. 2011, Astronomical Journal, 141, doi: 10.1088/0004-6256/141/3/70

Burgasser, A. J., Cruz, K. L., Cushing, M., et al. 2010, Astrophysical Journal, 710, 1142, doi: 10.1088/0004-637X/710/2/1142

Burgasser, A. J., Geballe, T. R., Leggett, S. K., Kirkpatrick, J. D., \& Golimowski, D. A. 2006, The Astrophysical Journal, 637, 1067, doi: 10.1086/498563

Burgasser, A. J., Kirkpatrick, J. D., Liebert, J., \& Burrows, A. 2003, The Astrophysical Journal, 594, 510, doi: 10.1086/376756

Burgasser, A. J., Looper, D. L., Kirkpatrick, J. D., Cruz, K. L., \& Swift, B. J. 2008b, The Astrophysical Journal, 674, 451, doi: 10.1086/524726

Burgasser, A. J., Looper, D. L., Kirkpatrick, J. D., \& Liu, M. C. 2007, The Astrophysical Journal, 658, 557, doi: $10.1086 / 511518$

Burgasser, A. J., \& McElwain, M. W. 2006, The Astronomical Journal, 131, 1007, doi: 10.1086/499042

Burgasser, A. J., McElwain, M. W., Kirkpatrick, J. D., et al. 2004, The Astronomical Journal, 127, 2856, doi: 10.1086/383549

Burrows, A., Hubbard, W. B., Lunine, J. I., \& Liebert, J. 2001, Reviews of Modern Physics, 73, 719, doi: 10.1103/RevModPhys.73.719

Burrows, A., Sudarsky, D., \& Hubeny, I. 2006, The Astrophysical Journal, 640, 1063 
Burrows, A., Sudarsky, D., \& Lunine, J. I. 2003, The Astrophysical Journal, 596, 587, doi: 10.1086/377709

Chabrier, G., \& Baraffe, I. 2000, Annual Review of Astronomy and Astrophysics, 38, 337

Charnay, B., Bézard, B., Baudino, J. L., et al. 2018, The Astrophysical Journal, 854, 172, doi: $10.3847 / 1538-4357 /$ aaac7d

Chiu, K., Fan, X., Leggett, S. K., et al. 2006, The Astronomical Journal, 131, 2722, doi: 10.1086/501431

Collaboration, G., Brown, A. G. A., Vallenari, A., et al. 2018, Astronomy and Astrophysics, 616, 1

Collaboration, G., Brown, A., Vallenari, A., et al. 2020, Astronomy and Astrophysics Astrophysics, 61, 1. https://arxiv.org/abs/2012.01533v1

Crepp, J. R., Principe, D. A., Wolff, S., et al. 2018, arXiv, 853, 192, doi: 10.3847/1538-4357/aaa2fd

Cruz, K. L., Burgasser, A. J., Reid, I. N., \& Liebert, J. 2004, The Astrophysical Journal, 604, L61

Cruz, K. L., Kirkpatrick, J. D., \& Burgasser, A. J. 2009, Astronomical Journal, 137, 3345, doi: 10.1088/0004-6256/137/2/3345

Cruz, K. L., Núñez, A., Burgasser, A. J., et al. 2017, The Astronomical Journal, 155, 34, doi: 10.3847/1538-3881/aa9d8a

Cushing, M. C., Saumon, D., \& Marley, M. S. 2010, Astronomical Journal, 140, 1428, doi: 10.1088/0004-6256/140/5/1428

Cushing, M. C., Roellig, T. L., Marley, M. S., et al. 2006, The Astrophysical Journal, 648, 614, doi: 10.1086/505637

Cushing, M. C., Marley, M. S., Saumon, D., et al. 2008, The Astrophysical Journal, 678, 1372, doi: 10.1086/526489

Cutri, R. M., Skrutskie, M. F., van Dyk, S., et al. 2003, 2MASS All Sky Catalog of point sources, Tech. rep., The IRSA 2MASS All-Sky Point Source Catalog, NASA/IPAC Infrared Science Archive. https://ui. adsabs.harvard.edu/\#abs/2003tmc..book.....C/abstract

Diolaiti, E., Bendinelli, O., Bonaccini, D., et al. 2000, Astronomy and Astrophysics Supplement Series, 147, 335, doi: 10.1051/aas:2000305

Dupuy, T. J., \& Liu, M. C. 2012, Astrophysical Journal, Supplement Series, 201, doi: 10.1088/0067-0049/201/2/19

- 2017, The Astrophysical Journal Supplement Series, 231, 15, doi: 10.3847/1538-4365/aa5e4c

Dupuy, T. J., Liu, M. C., \& Bowler, B. P. 2009, Astrophysical Journal, 706, 328, doi: 10.1088/0004-637X/706/1/328

Dupuy, T. J., Liu, M. C., \& Ireland, M. J. 2014, Astrophysical Journal, 790, doi: 10.1088/0004-637X/790/2/133
Faherty, J. K., Burgasser, A. J., Walter, F. M., et al. 2012, Astrophysical Journal, 752, doi: 10.1088/0004-637X/752/1/56

Faherty, J. K., Riedel, A. R., Cruz, K. L., et al. 2016, The Astrophysical Journal Supplement Series, 225, 10, doi: 10.3847/0067-0049/225/1/10

Ferguson, J. W., Alexander, D. R., Allard, F., et al. 2005, The Astrophysical Journal, 623, 585, doi: 10.1086/428642

Filippazzo, J. C., Rice, E. L., Faherty, J., et al. 2015, Astrophysical Journal, 810, doi: 10.1088/0004-637X/810/2/158

Freedman, R. S., Marley, M. S., \& Lodders, K. 2008, The Astrophysical Journal Supplement Series, 174, 504, doi: 10.1086/521793

Gao, P., Marley, M. S., \& Ackerman, A. S. 2018, The Astrophysical Journal, 855, 86, doi: 10.3847/1538-4357/aab0a1

Gelino, C. R., Smart, R. L., Marocco, F., et al. 2014, Astronomical Journal, 148, 4, doi: 10.1088/0004-6256/148/1/6

Gennaro, M., Baggett, S., \& Bajaj, V. 2019, A characterization of persistence at short times in the WFC3/IR detector. II, Tech. rep., STsCI. https://ui. adsabs.harvard.edu/\#abs/2018wfc..rept....5G/abstract

Gizis, J. E., Faherty, J. K., Liu, M. C., et al. 2012, Astronomical Journal, 144, 1, doi: 10.1088/0004-6256/144/4/94

Hauschildt, P. H. 1993, Journal of Quantitative Spectroscopy and Radiative Transfer, 50, 301, doi: https://doi.org/10.1016/0022-4073(93)90080-2

Hauschildt, P. H., Allard, F., \& Baron, E. 1998, The Astrophysical Journal, 512, 377, doi: 10.1086/306745

Hauschildt, P. H., \& Baron, E. 2006, Astronomy and Astrophysics, 451, 273, doi: 10.1051/0004-6361:20053846

Helling, C., \& Casewell, S. 2014, Astronomy and Astrophysics Review, 22, 1, doi: 10.1007/s00159-014-0080-0

Helling, C., Woitke, P., \& Thi, W. F. 2008a, Astronomy and Astrophysics, 485, 547, doi: 10.1051/0004-6361:20078220

Helling, C., Ackerman, A., Allard, F., et al. 2008b, Monthly Notices of the Royal Astronomical Society, 391, 1854, doi: 10.1111/j.1365-2966.2008.13991.x

Hiranaka, K., Cruz, K. L., Douglas, S. T., Marley, M. S., \& Baldassare, V. F. 2016, The Astrophysical Journal, 830, 1, doi: 10.3847/0004-637X/830/2/96

Kirkpatrick, J. D. 2005, Annual Review of Astronomy and Astrophysics, 43, 195, doi: 10.1146/annurev.astro.42.053102.134017 
Kirkpatrick, J. D., Cruz, K. L., Barman, T. S., et al. 2008, The Astrophysical Journal, 689, 1295, doi: 10.1086/592768

Kirkpatrick, J. D., Looper, D. L., Burgasser, A. J., et al. 2010, Astrophysical Journal, Supplement Series, 190, 100, doi: 10.1088/0067-0049/190/1/100

Knapp, G. R., Leggett, S. K., Fan, X., et al. 2004, The Astrophysical Journal, 3553

Konopacky, Q. M., Ghez, A. M., Barman, T. S., et al. 2010, Astrophysical Journal, 711, 1087, doi: 10.1088/0004-637X/711/2/1087

Krist, J. E., Hook, R. N., \& Stoehr, F. 2011, in Optical Modeling and Performance Predictions V, ed. M. A. Kahan, Vol. 8127, International Society for Optics and Photonics (SPIE), 166-181, doi: 10.1117/12.892762

Lane, B. F., Osorio, M. R. Z., Britton, M. C., Martin, E. L., \& Kulkarni, S. R. 2002, The Astrophysical Journal, 560, 390, doi: 10.1086/322506

Leggett, S. K., Golimowski, D. A., Fan, X., et al. 2002, The Astrophysical Journal, 564, 452, doi: 10.1086/324037

Lew, B. W. P., Apai, D., Zhou, Y., et al. 2016, The Astrophysical Journal Letters, 829, 1, doi: 10.3847/2041-8205/829/2/L32

Lew, B. W. P., Apai, D., Marley, M., et al. 2020, arXiv, doi: 10.3847/1538-4357/abb81d

Liu, M. C., Dupuy, T. J., \& Allers, K. N. 2016, The Astrophysical Journal, 833, 1, doi: 10.3847/1538-4357/833/1/96

Liu, M. C., Dupuy, T. J., \& Ireland, M. J. 2008, The Astrophysical Journal, 689, 436, doi: 10.1086/591837

Lodders, K., \& Fegley, B. 2002, Icarus, 155, 393, doi: $10.1006 /$ icar.2001.6740

Looper, D. L., Kirkpatrick, J. D., \& Burgasser, A. J. 2008a, The Astronomical Journal, 134, 1162, doi: 10.1086/520645

Looper, D. L., Kirkpatrick, J. D., Cutri, R. M., et al. 2008b, The Astrophysical Journal, 686, 528, doi: 10.1086/591025

Marley, M. S., Gelino, C., Stephens, D., Lunine, J. I., \& Freedman, R. 1999, The Astrophysical Journal, 513, 879, doi: 10.1086/306881

Marley, M. S., \& Robinson, T. D. 2015, Annual Review of Astronomy and Astrophysics, 53, 279, doi: 10.1146/annurev-astro-082214-122522

Marley, M. S., Saumon, D., \& Goldblatt, C. 2010, Astrophysical Journal Letters, 723, 117, doi: 10.1088/2041-8205/723/1/L117

Marley, M. S., Seager, S., Saumon, D., et al. 2002, The Astrophysical Journal, 568, 335, doi: 10.1086/338800
Marocco, F., Day-jones, A. C., Lucas, P. W., et al. 2014, Monthly Notices of the Royal Astronomical Society, 439, 372, doi: 10.1093/mnras/stt2463

Marois, C., Zuckerman, B., Konopacky, Q. M., Macintosh, B., \& Barman, T. 2010, Nature, 468, 1080, doi: $10.1038 /$ nature09684

McLean, I. S., McGovern, M. R., Burgasser, A. J., et al. 2003, The Astrophysical Journal, 596, 561, doi: 10.1086/377636

Metchev, S. A., \& Hillenbrand, L. A. 2006, The Astrophysical Journal, 651, 1166, doi: 10.1086/507836

Miles, B. E., Skemer, A. J. I., Morley, C. V., et al. 2020, The Astronomical Journal, 160, 63, doi: 10.3847/1538-3881/ab9114

Morley, C. V., Fortney, J. J., Marley, M. S., et al. 2012, Astrophysical Journal, 756, doi: 10.1088/0004-637X/756/2/172

Nakajima, T., Tsuji, T., \& Yanagisawa, K. 2004, The Astrophysical Journal, 607, 499, doi: 10.1086/383299

Radigan, J. 2014, Astrophysical Journal, 797, doi: 10.1088/0004-637X/797/2/120

Reid, I. N., Lewitus, E., Burgasser, A. J., \& Cruz, K. L. 2006, The Astrophysical Journal, 639, 1114, doi: 10.1086/499484

Saumon, D., Bergeron, P., Lunine, J. I., Hubbard, W. B., \& Burrows, A. 2012, The Astrophysical Journal, 424, 333

Saumon, D., \& Marley, M. S. 2008, The Astrophysical Journal, 689, 1327, doi: 10.1086/592734

Schmidt, S. J., West, A. A., Burgasser, A. J., Bochanski, J. J., \& Hawley, S. L. 2010, Astronomical Journal, 139, 1045, doi: 10.1088/0004-6256/139/3/1045

Sharp, C. M., \& Burrows, A. 2007, The Astrophysical Journal Supplement Series, 168, 140, doi: 10.1086/508708

Showman, A. P., Tan, X., \& Zhang, X. 2018, The Astronomical Journal, 883, 4, doi: 10.3847/1538-4357/ab384a

Stephens, D. C., Leggett, S. K., Cushing, M. C., et al. 2009, Astrophysical Journal, 702, 154, doi: 10.1088/0004-637X/702/1/154

Stone, J. M., Eisner, J., Skemer, A., et al. 2016, The Astrophysical Journal, 829, 39, doi: 10.3847/0004-637x/829/1/39

Stone, J. M., Barman, T., Skemer, A. J., et al. 2020, The Astronomical Journal, 160, 11. https://arxiv.org/abs/2010.02928

Tan, X., \& Showman, A. P. 2019, The Astrophysical Journal, 874, 111, doi: 10.3847/1538-4357/ab0c07

-. 2021a, Monthly Notices of the Royal Astronomical Society, 502, 2198, doi: 10.1093/mnras/stab097 
- 2021b, Monthly Notices of the Royal Astronomical Society, 502, 678, doi: 10.1093/mnras/stab060

Tennyson, J., \& Yurchenko, S. N. 2012, Monthly Notices of the Royal Astronomical Society, 425, 21, doi: 10.1111/j.1365-2966.2012.21440.x

Tsuji, T., \& Nakajima, T. 2003, The Astrophysical Journal, 151

Tsuji, T., Ohnaka, K., Aoki, W., \& Nakajima, T. 1996, Astronomy and Astrophysics, 308, L29.

https://arxiv.org/abs/arXiv:1011.1669v3

Wood, C. M., Boyajian, T., von Braun, K., et al. 2019, The Astrophysical Journal, 873, 83,

doi: 10.3847/1538-4357/aafe01
Zhang, X., \& Showman, A. P. 2014, Astrophysical Journal Letters, 788, 2, doi: 10.1088/2041-8205/788/1/L6

Zhang, Z., Liu, M. C., Marley, M. S., Line, M. R., \& Best, W. M. 2020, arXiv, 1. https://arxiv.org/abs/2011.12294

Zhou, Y., Apai, D., Metchev, S., et al. 2018, The Astronomical Journal, 155, 132,

doi: 10.3847/1538-3881/aaabbd 